\title{
Virtual braids
}

\author{
by \\ Louis H. Kauffman (Chicago, IL) and \\ Sofia Lambropoulou (Athens and Caen)
}

\begin{abstract}
This paper gives a new method for converting virtual knots and links to virtual braids. Indeed, the braiding method given here is quite general and applies to all the categories in which braiding can be accomplished. This includes the braiding of classical, virtual, flat, welded, unrestricted, and singular knots and links. We also give reduced presentations for the virtual braid group and for the flat virtual braid group (as well as for other categories). These reduced presentations are based on the fact that these virtual braid groups for $n$ strands are generated by a single braiding element plus the generators of the symmetric group on $n$ letters.
\end{abstract}

0. Introduction. Just as classical knots and links can be represented by the closures of braids, so can virtual knots and links be represented by the closures of virtual braids [16]. Virtual braids have a group structure that can be described by generators and relations [17], generalizing the generators and relations of the classical braid group. This structure of virtual braids is worth studying for its own sake. The virtual braid group is an extension of the classical braid group by the symmetric group. In [13] a Markov Theorem is proved for virtual braids, giving a set of moves on virtual braids that generate the same equivalence classes as the virtual link types of their closures. Such theorems are important for understanding the structure and classification of virtual knots and links.

In the present paper we give a new method for converting virtual knots and links to virtual braids. Indeed, the braiding method given in this paper is quite general and applies to all the categories in which braiding can be accomplished. This includes the braiding of classical, virtual, flat, welded, unrestricted, and singular knots and links. We also give reduced presentations for the virtual braid group and for the flat virtual braid group (as well as for other categories). These reduced presentations are based on the fact that these virtual braid groups for $n$ strands are generated by a single braiding element plus the generators of the symmetric group on $n$ letters.

2000 Mathematics Subject Classification: Primary 57M25. 
In a sequel to this paper we shall give a new way to establish the Markov Theorem for virtual braids via the $L$-move [22]. The $L$-move has provided a new approach to Markov Theorems in classical low dimensional topology, and it performs a similar role for virtual link theory. We shall recover the Markov Theorem of [13] and put it into the context of $L$-move theory. In that sequel paper we shall discuss the same issues for welded and flat virtual braids.

This paper consists in four sections. In Section 1 we review the definitions of virtual knot theory and flat virtual knot theory. We recall the interpretations of virtual knot theory in terms of abstract Gauss codes, and in terms of stabilized embeddings (immersions for flat virtuals) in thickened surfaces. We emphasize the role of the detour move and of the forbidden moves in the structure of the theory. A useful feature of this introduction is our description of ribbon neighborhood representations for virtual links and virtual braids. These representations (also called abstract link diagrams) give the least surface embedding (with boundary) that can represent a given link diagram. In Section 2 we give a new braiding algorithm for virtual braids. This algorithm generalizes Alexander's original algorithm, converting classical links to braid form. The present algorithm is quite general, and gives a uniform braiding method for many different categories. Section 3 gives the definition of the virtual braid group via generators and relations. We see the virtual braid group on $n$ strands, $V B_{n}$, as an extension of the classical braid group $B_{n}$ by the symmetric group $S_{n}$. The relationship between $S_{n}$ and $B_{n}$ in $V B_{n}$ is intricate. One remarkable property of these subgroups is that $V B_{n}$ can be generated by $S_{n}$ and a single generator of $B_{n}$ (a single twist, e.g. the braid $\sigma_{1}$ ). We give a reduced presentation of $V B_{n}$ that incorporates this reduction. In Section 4 we give a similar reduced presentation for the flat virtual braid group $F V_{n}$. The flat group $F V_{n}$ is a free product, with extra relations, of two copies of the symmetric group $S_{n}$. In Section 5 we detail reduced presentations for the welded braid group, the unrestricted virtual braid group and the flat unrestricted braid group. Section 6 concludes the paper with a topological interpretation of welded and flat unrestricted braids in terms of tubes embedded in four-space. All of these topics and formulations will be explored further in our subsequent papers.

For reference to previous work on virtual braids the reader should consult [5, 6, 9-14, 16-18, 20, 21, 23, 25-28]. For work on welded braids, see [7, 13]. For work on singular braids, see $[2,3,8,19,28]$. It is worth remarking that virtual braids embed in the Artin braids just as virtual knots embed in classical knots. This fact may be most easily deduced from [21], and can also be seen from [23] and [7]. We should also mention that this paper does not deal with invariants of braids, or with invariants of (virtual) knots and links that can be constructed by using braids. These topics will be dealt with in a sequel to the present paper. 
1. Virtual knot theory. Virtual knot theory is an extension of classical diagrammatic knot theory. In this extension one adds a virtual crossing (see Figure 1) that is neither an over-crossing nor an under-crossing. A virtual crossing is represented by two crossing arcs with a small circle placed around the crossing point.

Figure 1 illustrates the simplest example of a virtual knot. Note the appearance of a virtual crossing in the diagram. There is no way to represent in the plane the Gauss code of this diagram (shown in the figure) without entering a virtual crossing that is not registered in the code itself. Similar remarks apply to links and virtual links, where the codes are collections of sequences, one for each component of the link. One way to understand the structure of virtual knots and links is to regard their diagrams as planar representatives of possibly non-planar Gauss codes. The virtual crossings are artifacts of the planar representation.

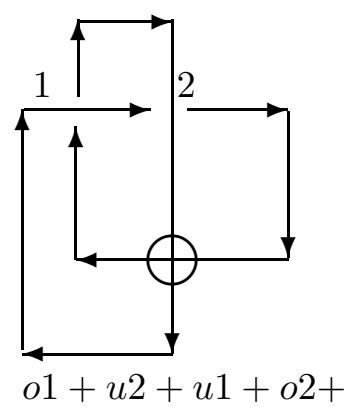

Fig. 1. Virtual knot and Gauss code

Moves on virtual diagrams generalize the Reidemeister moves for classical knot and link diagrams. See Figures 2 and 3. One can summarize the moves on virtual diagrams as follows: The classical crossings interact with one another according to the usual Reidemeister moves (Part A of Figure 2). The first move of Part A is called planar isotopy move. Virtual crossings interact with one another by Reidemeister moves that ignore the structure of under or over crossings (Part B of Figure 2). The key move between virtual and classical crossings is shown in Part $\mathrm{C}$ of Figure 2. Here a consecutive sequence of two virtual crossings can be moved across a single classical crossing. The moves containing virtual crossings (the moves in Part B and $\mathrm{C}$ of Figure 2) will be called virtual Reidemeister moves. All diagrammatic moves of Figure 2 are called augmented Reidemeister moves and they give rise to an equivalence relation in the set of virtual knot and link diagrams, called virtual equivalence or virtual isotopy or just isotopy.

The move in Part $\mathrm{C}$ of Figure 2 is a special case of the more general detour move indicated in Figure 3. We will call it the special detour move. In the detour move, an arc in the diagram that contains a consecutive sequence 


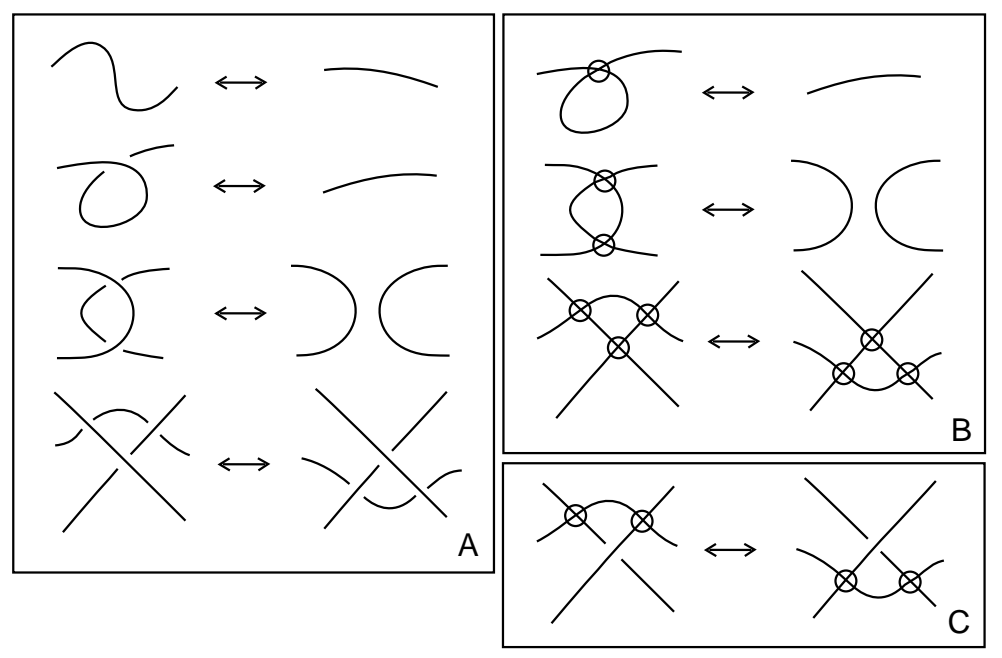

Fig. 2. Augmented Reidemeister moves for virtuals

of virtual crossings can be excised, and the arc re-drawn, transversal to the rest of the diagram (or itself), adding virtual crossings whenever these intersections occur. In fact, each of the moves in Parts B and C of Figure 2 can be regarded as special cases of the detour move of Figure 3.

The equivalence relation generated on virtual diagrams by virtual Reidemeister moves is the same as the equivalence relation on virtual diagrams generated by the detour move. To see this: Obviously the moves in B and $\mathrm{C}$ of Figure 2 are special cases of the detour move. On the other hand, by similar arguments to those in the classical Reidemeister Theorem, it follows that any detour move can be achieved by a finite sequence of local steps, each one being a virtual Reidemeister move. Thus the general detour move is itself the consequence of the collection of moves in Parts $\mathrm{B}$ and $\mathrm{C}$ of Figure 2.

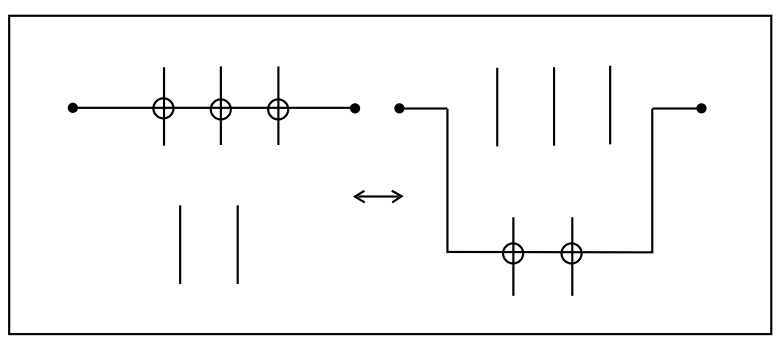

Fig. 3. The detour move 
A succinct description of virtual equivalence is that it is generated by classical Reidemeister moves and the detour move.

We note that a move analogous to the move in Part $\mathrm{C}$ of Figure 2 but with two real crossings and one virtual crossing is a forbidden move in virtual knot theory. There are two types of forbidden moves: One with an over arc, denoted $F_{1}$, and another with an under arc, denoted $F_{2}$. See [16] for explanations and interpretations. Variants of the forbidden moves are illustrated in Figure 4.
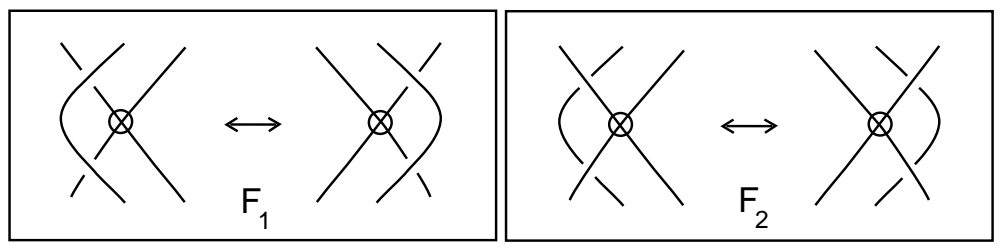

Fig. 4. The forbidden moves

We know $[16,9]$ that classical knot theory embeds faithfully in virtual knot theory. That is, if two classical knots are equivalent through moves using virtual crossings, then they are equivalent as classical knots via standard Reidemeister moves.

One can generalize many structures in classical knot theory to the virtual domain, and use the virtual knots to test the limits of classical problems such as the question whether the Jones polynomial detects knots. Counterexamples to this conjecture exist in the virtual domain. It is an open problem whether some of these counterexamples are isotopic to classical knots and links.

There is a useful topological interpretation for virtual knot theory in terms of embeddings of links in thickened surfaces. Regard each virtual crossing as a shorthand for a detour of one of the arcs in the crossing through a 1-handle that has been attached to the 2-sphere of the original diagram. By interpreting each virtual crossing in this way, we obtain an embedding of a collection of circles into a thickened surface $S_{g} \times I$, where $g$ is the number of virtual crossings in the original diagram, $S_{g}$ is a compact oriented surface of genus $g$ and $I$ denotes the unit interval. We say that two such surface embeddings are stably equivalent if one can be obtained from the other by isotopy in the thickened surfaces, homeomorphisms of the surfaces and the addition or subtraction of empty handles. Then we have the following.

TheOREm $([16,18,5])$. Two virtual link diagrams are equivalent if and only if their corresponding surface embeddings are stably equivalent.

See 1.2 below for more discussion of surfaces and virtuals. 
1.1. Flat virtual knots and links. Every classical knot or link diagram can be regarded as an immersion of cirlces in the plane with extra structure at the double points. This extra structure is usually indicated by the over and under crossing conventions that give instructions for constructing an embedding of the link in three-dimensional space from the diagram. If we take the diagram without this extra structure, it is the shadow of some link in three-dimensional space, but the weaving of that link is not specified. We call these shadow crossings flat crossings. It is well known that if one is allowed to apply the Reidemeister moves to such a shadow (without regard to the types of crossing since they are not specified) then the shadow can be reduced to a disjoint union of circles. This reduction is no longer true in the presence of virtual crossings.

More precisely, let a flat virtual diagram be a diagram with virtual crossings and flat crossings. Two flat virtual diagrams are equivalent if there is a sequence of generalized flat Reidemeister moves taking one to the other. A generalized flat Reidemeister move is any move as shown in Figure 2, but with flat crossings in place of classical crossings. Note that in studying flat virtuals the rules for changing virtual crossings among themselves and the rules for changing flat crossings among themselves are identical. However, detour moves as in Figure 2C are available only for virtual crossings with respect to flat crossings and not the other way around. The study of flat virtual knots and links was initiated in [16]. The category of flat virtual knots is identical in structure to what are called virtual strings by V. Turaev in $[26]$.

We shall say that a virtual diagram overlies a flat diagram if the virtual diagram is obtained from the flat diagram by choosing a crossing type for each flat crossing in the virtual diagram. To each virtual diagram $K$ there is an associated flat diagram $F(K)$ that is obtained by forgetting the extra structure at the classical crossings in $K$. Note that if $K$ is equivalent to $K^{\prime}$ as virtual diagrams, then $F(K)$ is equivalent to $F\left(K^{\prime}\right)$ as flat virtual diagrams. Thus, if we can show that $F(K)$ is not reducible to a disjoint union of circles, then it will follow that $K$ is a non-trivial virtual link.

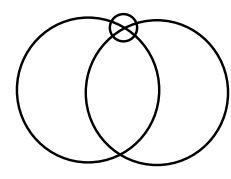

$\mathrm{H}$

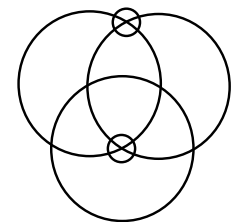

$\mathrm{L}$

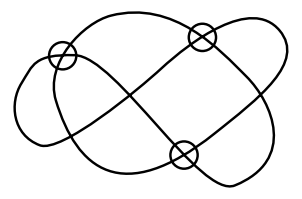

D

Fig. 5. Examples of flat knots and links 
Figure 5 ilustrates flat virtual links $H$ and $L$ and a flat virtual knot $D$. The link $H$ cannot be undone in the flat category because it has an odd number of virtual crossings between its two components and each generalized Reidemeister move preserves the parity of the number of virtual crossings between components. The diagram $D$ is shown to be a non-trivial flat virtual knot using the filamentation invariant that is introduced in [10]. The diagram $L$ is also a non-trivial flat diagram. Note that it comes apart at once if we allow the forbidden move.

The flat virtual diagrams present a strong challenge for the construction of new invariants. It is important to understand the structure of flat virtual knots and links. This structure lies at the heart of the comparison of classical and virtual links. We wish to be able to determine when a given virtual link is equivalent to a classical link. The reducibility or irreducibility of the underlying flat diagram is the first obstruction to such an equivalence. See $[10,11,26]$ for a discussion of combinatorial invariants of flat virtual knots based on the underlying Gauss code.

Just as virtual knots and links can be interpreted via stabilized embeddings of curves in thickened surfaces, flat virtuals can be interpreted as stabilized immersions of curves in surfaces (no thickening required). See [12] for applications of this point of view.

1.2. Ribbon neighborhood representations. As we have indicated above, virtual knots and links can be represented as knots and links in thickened surfaces. Another way to make this representation is to form a ribbon neighborhood surface (sometimes called an abstract link diagram [14]) for a given virtual knot or link, as illustrated in Figure 6. In this figure we show how a virtual trefoil knot (two classical and one virtual crossing) has the classical crossings represented as diagrammatic crossings in disks, which are connected by ribbons, while the virtual crossings are represented by ribbons that pass over one another without interacting. The abstract link diagram is shown embedded in three-dimensional space, but it is to be regarded as specified without any particular embedding. Thus it can be represented with the ribbons for the virtual crossings switched.

The abstract link diagram is a method for representing a virtual diagram (as an embedding in a thickened surface) that is distinct from our description in terms of handles given just before 1.1. These two points of view can be
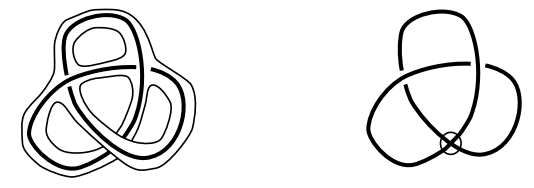

Fig. 6. A ribbon neighborhood surface for the virtual trefoil 


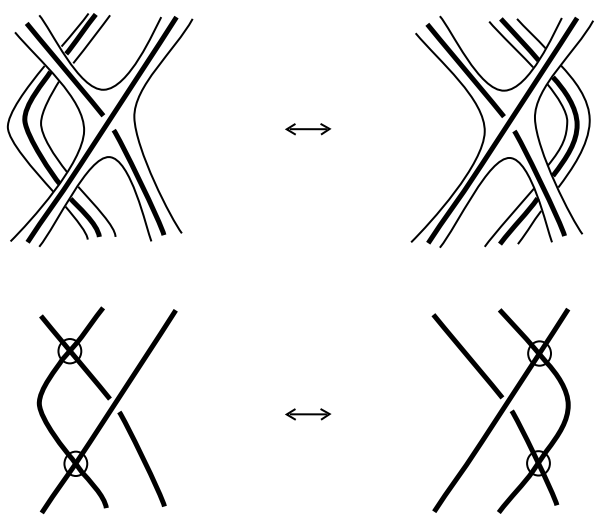

Fig. 7. A special detour move

related to one another, and this will be done elsewhere. Here we note that in the abstract link diagram any closed boundaries can be filled in with disks or with any convenient orientable surface with boundary. This is in accord with the representation of virtual knots and links as embeddings in thickened surfaces, taken up to addition and subtraction of empty handles.

In Figure 7 we illustrate the abstract link diagram for one of the special detour moves for virtuals (in braided form). Note again how this detour move is accomplished via the freedom of movement of the virtual crossings represented by non-interacting ribbons.
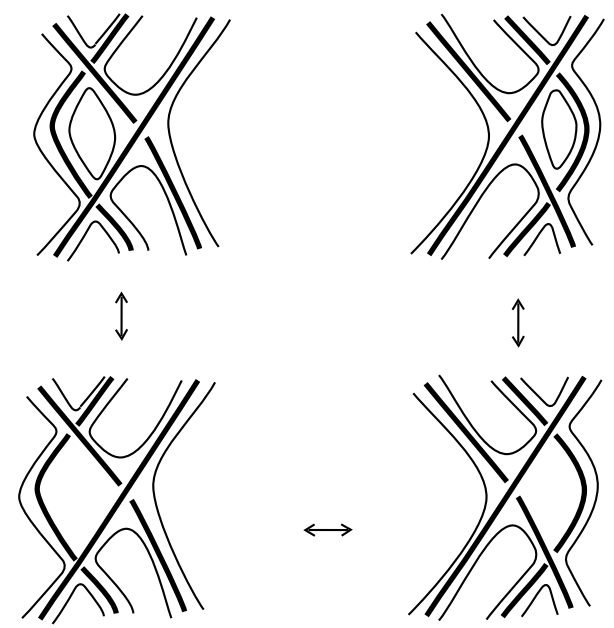

Fig. 8. Ribbon version of third Reidemeister move 


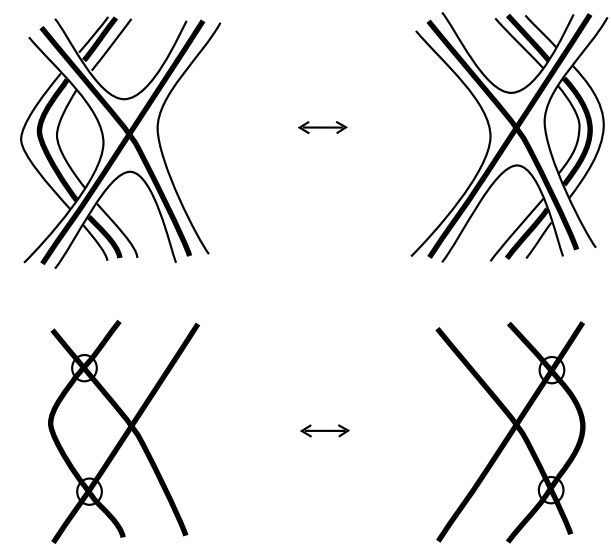

Fig. 9. Flat version of the detour move

In Figure 8 we illustrate a variant of the classical third Reidemeister move in surface form. Note that we accomplish this move by adding a disk and then performing an isotopy of the diagram on the surface.

Finally, in Figure 9 we illustrate the special detour move for flat virtuals using abstract link diagrams. Note the stark difference here between the virtual crossing structure and the immersion structure of the flat crossings.

2. Braiding link diagrams. A virtual braid on $n$ strands is a braid on $n$ strands in the classical sense, which may also contain virtual crossings. The closure of a virtual braid is obtained by joining with simple arcs the corresponding endpoints of the braid. The set of isotopy classes of virtual braids on $n$ strands forms a group. The virtual braid group structure will be defined in the next section. In this section we shall describe a simple and general method for converting any virtual knot or link diagram into the closure of a virtual braid. That is, we shall give a new proof (see [13]) that the classical Alexander Theorem [1,4] generalizes to virtuals and, in fact, to all the categories in which braids are constructed.

THEOREM 1. Every (oriented) virtual link can be represented by a virtual braid whose closure is isotopic to the original link.

Proof. The context of our braiding method is based on [22]. Any virtual link diagram can be arranged to be in general position with respect to the standard height function on the plane. This means that it does not contain any horizontal arcs and it can be seen as a composition of horizontal stripes, each containing either a local minimum or a local maximum or a crossing (of classical or virtual type). 


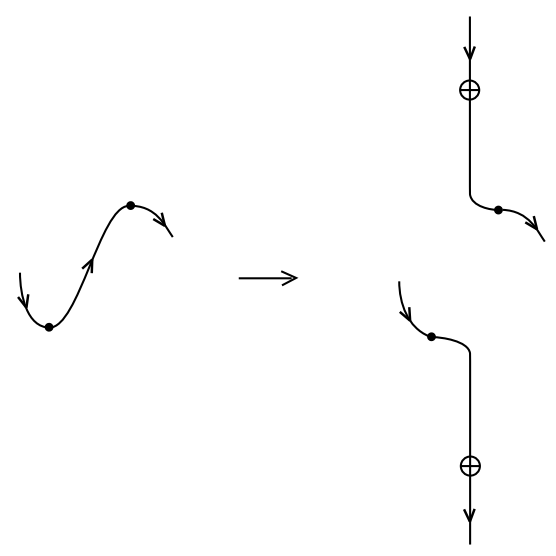

Fig. 10. The braiding move

The idea of the braiding is on the one hand to keep the down-arcs of the diagram that are oriented downwards and on the other hand to eliminate the up-arcs that go upwards, and produce instead braid strands. First consider up-arcs that occur between maxima and minima and contain no crossings. Call such an arc in the diagram a free up-arc.

We eliminate a free up-arc as follows: We cut the arc at a point. We then pull the two arcs, the upper upward and the lower downward, keeping their ends aligned, and so as to obtain a pair of corresponding braid strands, which create only virtual crossings with the rest of the diagram. See Figure 10. This operation will be called a braiding move. The closure of the resulting tangle is a virtual link diagram, obviously isotopic to the original one. Indeed, from the free up-arc we created a stretched loop, which by the detour move is isotopic to the up-arc.

Before performing these braiding moves, we prepare the diagram by rotating all the crossings so that any arcs that pass through the crossings are directed downwards. There are two types of rotation: If only one arc in the crossing goes up, then we rotate the crossing by 90 degrees. If both arcs of the crossing go up, then we rotate it by 180 degrees. See Figures 11 and 12.

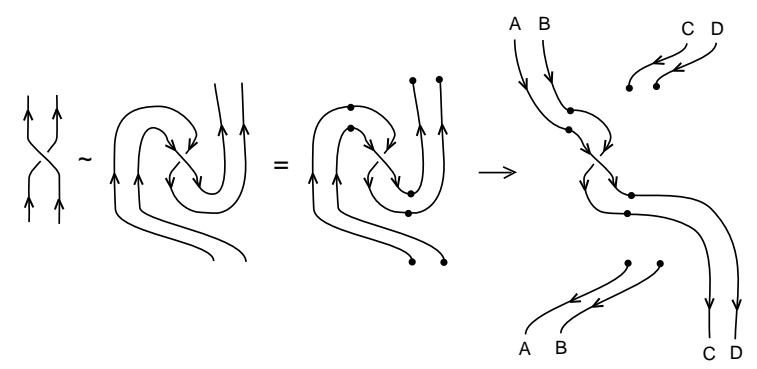

Fig. 11. Full twist 

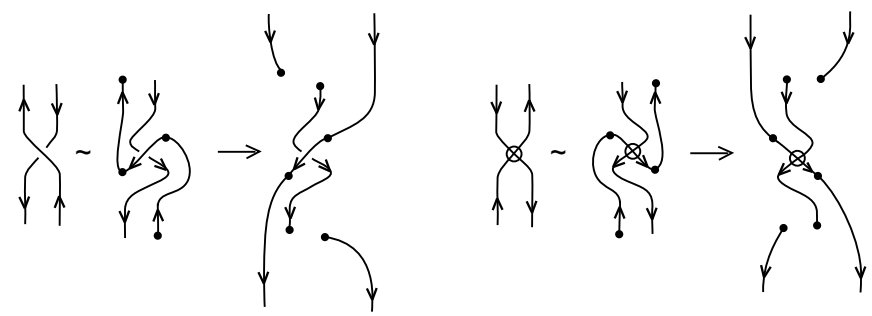

Fig. 12. Half twist

These rotations may produce new free up-arcs. After adjusting all the crossings, we then braid all the free up-arcs. The resulting tangle is the desired virtual braid, the closure of which is isotopic to the original diagram.

The braiding algorithm given above will braid any virtual diagram and thus it proves the analogue of the Alexander Theorem for virtual links.

In Figures 13 and 14 we illustrate an example. In Figure 13 we show a virtual knot and its preparation for braiding by crossing rotation. In Figure 14 we braid the arcs of the diagram prepared in Figure 13. In the intermediate stage of Figure 14, we break each up-arc, and we name each pair of endpoints with the same letter.
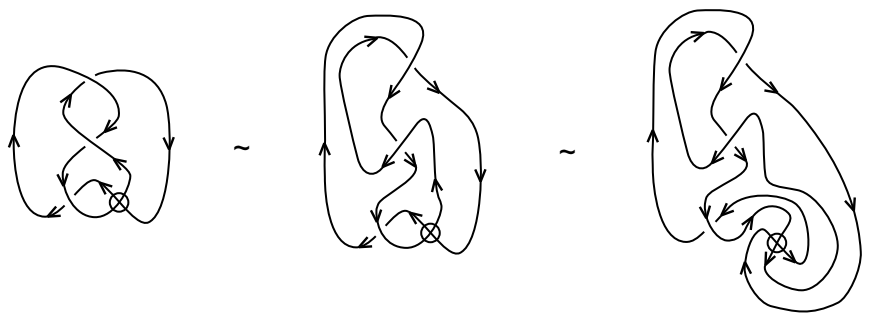

Fig. 13. Prepare example
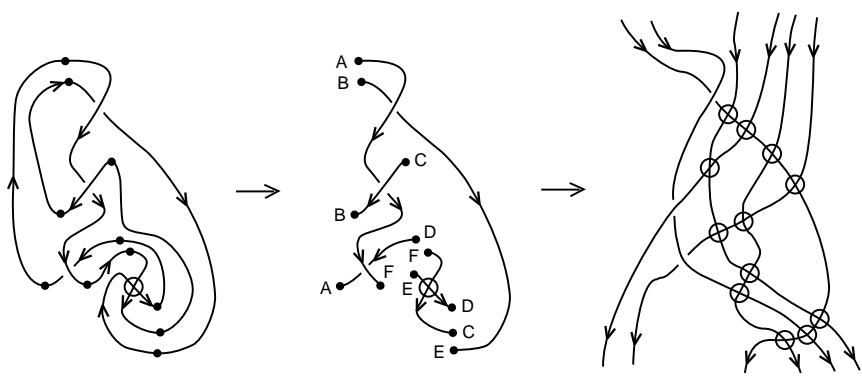

Fig. 14. Braid example

REMARK 1. The braiding technique, described in this section, applies equally well to flat virtual braids with no change in the description of the 
procedure. (See Section 4 for definition and further discussion on the flat braid group.) For welded and unrestricted virtual braids the procedure is the same with welded crossings replacing the role of virtual crossings. (See Section 5 for definition and further discussion on the corresponding braid group structures.) To use this procedure to create a classical braid from a classical knot or link diagram, the braiding of arcs must be done so that the new braid strands run entirely over the previously constructed tangle or entirely under it. The same procedure applies also for singular braids $[2,3]$ with singular crossings replacing the role of classical crossings. In this way, this braiding method works in all the categories in which braids are constructed.

3. A reduced presentation for the virtual braid group. The set of isotopy classes of virtual braids on $n$ strands forms a group, the virtual braid group denoted $V B_{n}$, that was introduced in [16]. The group operation is the usual braid multiplication (form $b b^{\prime}$ by attaching the bottom strand ends of $b$ to the top strand ends of $\left.b^{\prime}\right) . V B_{n}$ is generated by the usual braid generators $\sigma_{1}, \ldots, \sigma_{n-1}$ and by the virtual generators $v_{1}, \ldots, v_{n-1}$, where each virtual crossing $v_{i}$ has the form of the braid generator $\sigma_{i}$ with the crossing replaced by a virtual crossing. See Figure 15 for illustrations. Recall that in virtual crossings we do not distinguish between under and over crossing. Thus, $V B_{n}$ is an extension of the classical braid group $B_{n}$ by the symmetric group $S_{n}$.

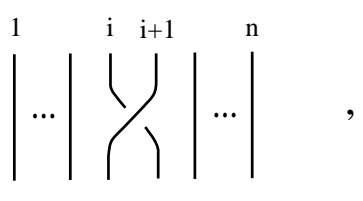

$\sigma_{i}$

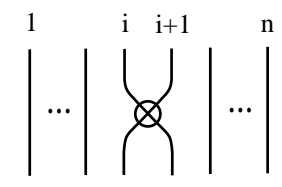

$v_{i}$

Fig. 15. The generators of $V B_{n}$

Among themselves the braid generators satisfy the usual braiding relations:

$$
\sigma_{i} \sigma_{i+1} \sigma_{i}=\sigma_{i+1} \sigma_{i} \sigma_{i+1}, \quad \sigma_{i} \sigma_{j}=\sigma_{j} \sigma_{i} \quad \text { for } j \neq i \pm 1 .
$$

Among themselves, the virtual generators are a presentation for the group $S_{n}$, so they satisfy the following virtual relations:

$$
\begin{aligned}
v_{i}^{2} & =1, \\
v_{i} v_{i+1} v_{i} & =v_{i+1} v_{i} v_{i+1} \\
v_{i} v_{j} & =v_{j} v_{i} \quad \text { for } j \neq i \pm 1 .
\end{aligned}
$$

It is worth noting at this point that the virtual braid group $V B_{n}$ does not embed in the classical braid group $B_{n}$, since the virtual braid group contains torsion elements and it is well known that $B_{n}$ does not. The mixed relations 
between virtual generators and braiding generators are as follows:

$$
\sigma_{i} v_{j}=v_{j} \sigma_{i} \quad \text { for } j \neq i \pm 1, \quad v_{i} \sigma_{i+1} v_{i}=v_{i+1} \sigma_{i} v_{i+1} .
$$

The second mixed relation will be called the special detour relation and it is illustrated in Figure 7. Note that the following relations are also special detour moves for virtual braids and they are easy consequences of the above:

$$
\begin{aligned}
\sigma_{i}^{-1} v_{i+1} v_{i} & =v_{i+1} v_{i} \sigma_{i+1}^{-1}, \\
v_{i} v_{i+1} \sigma_{i}^{ \pm} & =\sigma_{i+1}^{ \pm} v_{i} v_{i+1}, \\
\sigma_{i}^{ \pm} v_{i+1} v_{i} & =v_{i+1} v_{i} \sigma_{i+1}^{ \pm} .
\end{aligned}
$$

This set of relations taken together define the basic isotopies for virtual braids. Note that each relation is a braided version of a local virtual link isotopy. The special detour relation is written equivalently as $\sigma_{i+1}=v_{i} v_{i+1} \sigma_{i} v_{i+1} v_{i}$. Notice that this relation is the braid detour move of the $i$ th strand around the crossing between the $(i+1)$ st and the $(i+2)$ nd strand (see illustration in Figure 16) and it provides an inductive way of expressing all braiding generators in terms of the first braiding generator $\sigma_{1}$ and the virtual generators $v_{1}, \ldots, v_{n-1}$.

In this section we give a reduced presentation for $V B_{n}$ with generators

$$
\left\{\sigma_{1}, v_{1}, \ldots, v_{n-1}\right\}
$$

where we assume the defining relations:

$$
\sigma_{i+1}:=\left(v_{i} \ldots v_{2} v_{1}\right)\left(v_{i+1} \ldots v_{3} v_{2}\right) \sigma_{1}\left(v_{2} v_{3} \ldots v_{i+1}\right)\left(v_{1} v_{2} \ldots v_{i}\right)
$$

for $i=1, \ldots, n-2$. In terms of braid diagrams, this relation is the braid detour move of the strands $1,2, \ldots, i$ around the crossing $\sigma_{i+1}$. See right hand illustration in Figure 16.

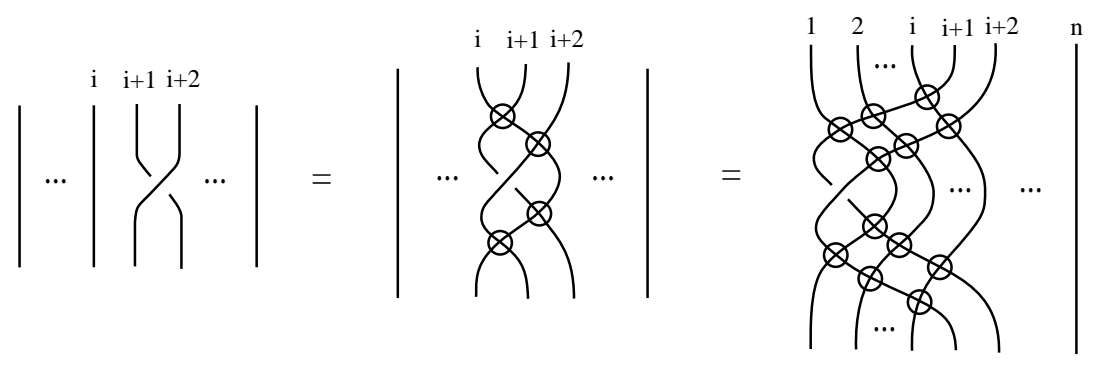

Fig. 16. Detouring the crossing $\sigma_{i+1}$

REMARK 2. By the detour move, in the same way that a crossing can be detoured to the first position of the braid, similarly any box in the braid can be detoured to the first position (in fact, to any position); see Figure 17. There it may undergo some changes and then it can be detoured back to its original position in the braid. In particlular, a relation in $V B_{n}$ occurring in 
a box in the interior of a braid may be redundant. In order to omit it we detour the box to the first position, there we apply a specific relation (see statement of Theorem 2) and then we detour the result back, thus obtaining the other side of the relation that we wanted to omit.

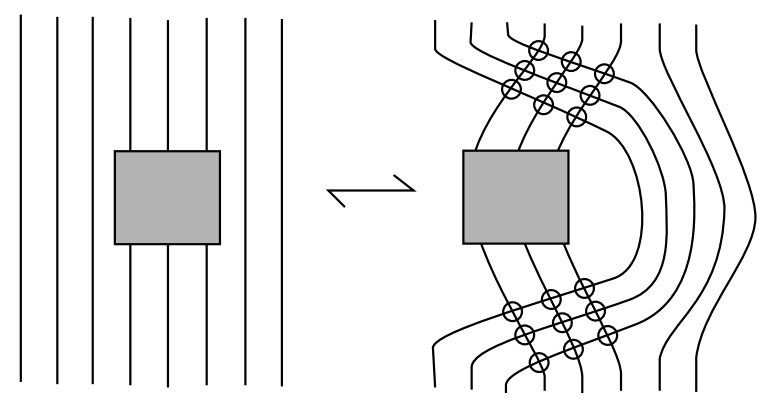

Fig. 17. Detouring a box

THEOREM 2. The virtual braid group $V B_{n}$ has the following reduced presentation:

$$
\begin{gathered}
V B_{n}=\left\langle\sigma_{1}, v_{1}, \ldots, v_{n-1}\right| v_{i} v_{i+1} v_{i}=v_{i+1} v_{i} v_{i+1}, v_{i} v_{j}=v_{j} v_{i}, j \neq i \pm 1, \\
v_{i}^{2}=1,1 \leq i \leq n-1, \sigma_{1} v_{j}=v_{j} \sigma_{1}, j>2, \\
\left(v_{1} \sigma_{1} v_{1}\right)\left(v_{2} \sigma_{1} v_{2}\right)\left(v_{1} \sigma_{1} v_{1}\right)=\left(v_{2} \sigma_{1} v_{2}\right)\left(v_{1} \sigma_{1} v_{1}\right)\left(v_{2} \sigma_{1} v_{2}\right), \\
\left.\sigma_{1}\left(v_{2} v_{3} v_{1} v_{2} \sigma_{1} v_{2} v_{1} v_{3} v_{2}\right)=\left(v_{2} v_{3} v_{1} v_{2} \sigma_{1} v_{2} v_{1} v_{3} v_{2}\right) \sigma_{1}\right\rangle .
\end{gathered}
$$

In Figure 18 we illustrate the last two reduced relations.

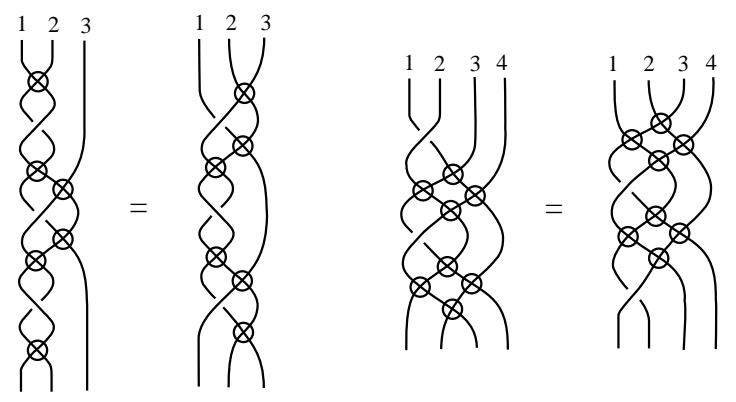

Fig. 18. The main reduced relations

Note that the relation

$$
\left(v_{1} \sigma_{1} v_{1}\right)\left(v_{2} \sigma_{1} v_{2}\right)\left(v_{1} \sigma_{1} v_{1}\right)=\left(v_{2} \sigma_{1} v_{2}\right)\left(v_{1} \sigma_{1} v_{1}\right)\left(v_{2} \sigma_{1} v_{2}\right)
$$

is equivalent to the relation

$$
\sigma_{1}\left(v_{1} v_{2} \sigma_{1} v_{2} v_{1}\right) \sigma_{1}=\left(v_{1} v_{2} \sigma_{1} v_{2} v_{1}\right) \sigma_{1}\left(v_{1} v_{2} \sigma_{1} v_{2} v_{1}\right),
$$


which reflects the braid relation $\sigma_{1} \sigma_{2} \sigma_{1}=\sigma_{2} \sigma_{1} \sigma_{2}$. Also, the relation

$$
\sigma_{1}\left(v_{2} v_{3} v_{1} v_{2} \sigma_{1} v_{2} v_{1} v_{3} v_{2}\right)=\left(v_{2} v_{3} v_{1} v_{2} \sigma_{1} v_{2} v_{1} v_{3} v_{2}\right) \sigma_{1}
$$

reflects the braid relation $\sigma_{1} \sigma_{3}=\sigma_{3} \sigma_{1}$. Therefore:

- From the commuting relations $\sigma_{i} \sigma_{j}=\sigma_{j} \sigma_{i}$ we only need to keep $\sigma_{1} \sigma_{3}=\sigma_{3} \sigma_{1}$.

- From the relations $\sigma_{i} \sigma_{i+1} \sigma_{i}=\sigma_{i+1} \sigma_{i} \sigma_{i+1}$ we only need to keep $\sigma_{1} \sigma_{2} \sigma_{1}=\sigma_{2} \sigma_{1} \sigma_{2}$.

Proof of Theorem 2. We note first that the special detour relations $v_{i} \sigma_{i+1} v_{i}=v_{i+1} \sigma_{i} v_{i+1}$ can be omitted in the reduced presentation, since they were used in the new defining relations $(*)$ for the $\sigma_{j}$ 's. The proof of the reduced presentation is then a consequence of the three lemmas below. The proofs of these lemmas are based on the simple geometric idea described in Remark 2. In the proofs we underline the expressions that are used each time in the next step.

LEMma 1. The mixed relations $\sigma_{i} v_{j}=v_{j} \sigma_{i}$ for $i>1$ and $j \neq i \pm 1$ follow from the defining relations $(*)$, the virtual relations and the reduced relations $\sigma_{1} v_{j}=v_{j} \sigma_{1}$ for $j>2$.

Proof. Substituting we have:

$$
\sigma_{i} v_{j} \stackrel{(*)}{=}\left(v_{i-1} \ldots v_{2} v_{1}\right)\left(v_{i} \ldots v_{3} v_{2}\right) \sigma_{1}\left(v_{2} v_{3} \ldots v_{i}\right)\left(v_{1} v_{2} \ldots v_{i-1}\right) v_{j} .
$$

Since $j \neq i \pm 1$ either $j \geq i+2$ or $j \leq i-2$. If $j \geq i+2$, then in the above expression $v_{j}$ clearly commutes with all generators, thus $\sigma_{i} v_{j}=v_{j} \sigma_{i}$. If $j \leq i-2$ we have

$$
\begin{aligned}
& \sigma_{i} v_{j}=\left(v_{i-1} \ldots v_{2} v_{1}\right)\left(v_{i} \ldots v_{3} v_{2}\right) \sigma_{1}\left(v_{2} v_{3} \ldots v_{i}\right)\left(v_{1} v_{2} \ldots v_{i-1}\right) \underline{v_{j}} \\
& =\left(v_{i-1} \ldots v_{2} v_{1}\right)\left(v_{i} \ldots v_{3} v_{2}\right) \sigma_{1}\left(v_{2} v_{3} \ldots v_{i}\right) \\
& \cdot\left(v_{1} v_{2} \ldots v_{j-1} v_{j} v_{j+1} v_{j} v_{j+2} \ldots v_{i-1}\right) \\
& =\left(v_{i-1} \ldots v_{2} v_{1}\right)\left(v_{i} \ldots v_{3} v_{2}\right) \sigma_{1}\left(v_{2} v_{3} \ldots v_{j+1} v_{j+2} \ldots v_{i}\right) \\
& \cdot\left(v_{1} v_{2} \ldots v_{j-1} v_{j+1} v_{j} v_{j+1} v_{j+2} \ldots v_{i-1}\right) \\
& =\left(v_{i-1} \ldots v_{2} v_{1}\right)\left(v_{i} \ldots v_{3} v_{2}\right) \sigma_{1}\left(v_{2} v_{3} \ldots v_{j} \underline{v_{j+1} v_{j+2} v_{j+1}} v_{j+3} \ldots v_{i}\right) \\
& \cdot\left(v_{1} v_{2} \ldots v_{i-1}\right) \\
& =\left(v_{i-1} \ldots v_{2} v_{1}\right)\left(v_{i} \ldots v_{j+2} v_{j+1} v_{j} \ldots v_{3} v_{2}\right) \sigma_{1} \\
& \cdot\left(v_{2} v_{3} \ldots v_{j} v_{j+2} v_{j+1} v_{j+2} v_{j+3} \ldots v_{i}\right)\left(v_{1} v_{2} \ldots v_{i-1}\right) \\
& =\left(v_{i-1} \ldots v_{2} v_{1}\right)\left(v_{i} \ldots v_{j+3} \underline{v_{j+2} v_{j+1} v_{j+2}} v_{j} \ldots v_{3} v_{2}\right) \sigma_{1}\left(v_{2} v_{3} \ldots v_{i}\right) \\
& \text { - }\left(v_{1} v_{2} \ldots v_{i-1}\right)
\end{aligned}
$$




$$
\begin{aligned}
= & \left(v_{i-1} \ldots v_{j+1} v_{j} v_{j-1} \ldots v_{2} v_{1}\right)\left(v_{i} \ldots v_{j+3} \underline{v_{j+1}} v_{j+2} v_{j+1} v_{j} \ldots v_{3} v_{2}\right) \\
& \cdot \sigma_{1}\left(v_{2} v_{3} \ldots v_{i}\right)\left(v_{1} v_{2} \ldots v_{i-1}\right) \\
= & \left(v_{i-1} \ldots v_{j+2} \underline{v_{j+1} v_{j} v_{j+1}} v_{j-1} \ldots v_{2} v_{1}\right)\left(v_{i} \ldots v_{3} v_{2}\right) \sigma_{1}\left(v_{2} v_{3} \ldots v_{i}\right) \\
& \cdot\left(v_{1} v_{2} \ldots v_{i-1}\right) \\
= & \left(v_{i-1} \ldots v_{j+2} \underline{v_{j}} v_{j+1} v_{j} v_{j-1} \ldots v_{2} v_{1}\right)\left(v_{i} \ldots v_{3} v_{2}\right) \sigma_{1}\left(v_{2} v_{3} \ldots v_{i}\right) \\
& \cdot\left(v_{1} v_{2} \ldots v_{i-1}\right) \\
= & v_{j}\left(v_{i-1} \ldots v_{2} v_{1}\right)\left(v_{i} \ldots v_{3} v_{2}\right) \sigma_{1}\left(v_{2} v_{3} \ldots v_{i}\right)\left(v_{1} v_{2} \ldots v_{i-1}\right) \\
\stackrel{(*)}{=} & v_{j} \sigma_{i} .
\end{aligned}
$$

In the proofs of Lemmas 2 and 3 below we use repeatedly the following virtual braid relations, which are easy consequences of the virtual relations:

(†) $\quad\left(v_{4} v_{3} v_{2} v_{1}\right) \ldots\left(v_{i+2} v_{i+1} v_{i} v_{i-1}\right)$

$$
=\left(v_{4} \ldots v_{i+2}\right)\left(v_{3} \ldots v_{i+1}\right)\left(v_{2} \ldots v_{i}\right)\left(v_{1} \ldots v_{i-1}\right) \text {. }
$$

LEMMA 2. The braid relations $\sigma_{i} \sigma_{i+1} \sigma_{i}=\sigma_{i+1} \sigma_{i} \sigma_{i+1}$ for $i>1$ follow from the defining relations $(*)$, the virtual relations, the reduced relations $\sigma_{1} v_{j}=v_{j} \sigma_{1}$ of Lemma 1 , and the reduced relation

$$
\sigma_{1}\left(v_{1} v_{2} \sigma_{1} v_{2} v_{1}\right) \sigma_{1}=\left(v_{1} v_{2} \sigma_{1} v_{2} v_{1}\right) \sigma_{1}\left(v_{1} v_{2} \sigma_{1} v_{2} v_{1}\right)
$$

Proof. Indeed, on the one hand we have

$$
\begin{aligned}
& \sigma_{i} \sigma_{i+1} \sigma_{i} \stackrel{(*)}{=}\left[( v _ { i - 1 } \ldots v _ { 1 } ) ( v _ { i } \ldots v _ { 2 } ) \sigma _ { 1 } ( v _ { 2 } \ldots v _ { i } ) \left(\underline{\left.\left.v_{1} \ldots v_{i-1}\right)\right]\left[\left(v_{i} v_{i-1} \ldots v_{1}\right)\right.}\right.\right. \\
& \left.\cdot\left(v_{i+1} \ldots v_{2}\right) \sigma_{1}\left(v_{2} \ldots v_{i+1}\right)\left(v_{1} \ldots v_{i-1} v_{i}\right)\right]\left[\left(v_{i-1} \ldots v_{1}\right)\right. \\
& \left.\cdot\left(v_{i} \ldots v_{2}\right) \sigma_{1}\left(v_{2} \ldots v_{i}\right)\left(v_{1} \ldots v_{i-1}\right)\right] \\
& \stackrel{(\dagger)}{=}\left(v_{i-1} \ldots v_{1}\right)\left(v_{i} \ldots v_{2}\right) \sigma_{1}\left(v_{2} \ldots v_{i}\right)\left(v_{i} \ldots v_{2} v_{1} v_{2} \ldots v_{i}\right)\left(v_{i+1} \ldots v_{2}\right) \sigma_{1} \\
& \cdot\left(v_{2} \ldots v_{i+1}\right)\left(v_{i} \ldots v_{2} v_{1} \underline{\left.v_{2} \ldots v_{i}\right)\left(v_{i} \ldots v_{2}\right)} \sigma_{1}\left(v_{2} \ldots v_{i}\right)\left(v_{1} \ldots v_{i-1}\right)\right. \\
& =\left(v_{i-1} \ldots v_{1}\right)\left(v_{i} \ldots v_{2}\right) \sigma_{1}\left(v_{1} v_{2} \ldots v_{i}\right)\left(v_{i+1} v_{i} \ldots v_{2}\right) \sigma_{1} \\
& \left.\cdot\left(\underline{\left.v_{2} \ldots v_{i} v_{i+1}\right)\left(v_{i} \ldots v_{2}\right.} v_{1}\right) \overline{\sigma_{1}\left(v_{2} \ldots v_{i}\right)\left(v_{1} \ldots v_{i-1}\right.}\right) \\
& \stackrel{(\dagger)}{=}\left(v_{i-1} \ldots v_{1}\right)\left(v_{i} \ldots v_{2}\right) \sigma_{1} v_{1}\left(\underline{v_{i+1} \ldots v_{3}} v_{2} v_{3} \ldots v_{i+1}\right) \underline{\sigma_{1}} \\
& \cdot\left(v_{i+1} \ldots v_{3} v_{2} v_{3} \ldots v_{i+1}\right) \underline{v_{1} \sigma_{1}}\left(v_{2} \ldots v_{i}\right)\left(v_{1} \ldots v_{i-1}\right) \\
& =\left(v_{i-1} \ldots v_{1}\right)\left(v_{i} \ldots v_{2}\right)\left(v_{i+1} \ldots v_{3}\right) \sigma_{1} v_{1} v_{2} \sigma_{1}\left(v_{3} \ldots v_{i+1}\right)\left(v_{i+1} \ldots v_{3}\right) \\
& \text { - } v_{2} v_{1} \sigma_{1}\left(v_{3} \ldots v_{i+1}\right)\left(v_{2} \ldots v_{i}\right)\left(v_{1} \ldots v_{i-1}\right) \\
& =\left(v_{i-1} \ldots v_{1}\right)\left(v_{i} \ldots v_{2}\right)\left(v_{i+1} \ldots v_{3}\right) \underline{\sigma_{1} v_{1} v_{2} \sigma_{1} v_{2} v_{1} \sigma_{1}}\left(v_{3} \ldots v_{i+1}\right) \\
& \cdot\left(v_{2} \ldots v_{i}\right)\left(v_{1} \ldots v_{i-1}\right)
\end{aligned}
$$




$$
\begin{aligned}
= & \left(v_{i-1} \ldots v_{1}\right)\left(v_{i} \ldots v_{2}\right)\left(v_{i+1} \ldots v_{3}\right) \underline{v_{1}} v_{2} \sigma_{1} v_{2} v_{1} \sigma_{1} v_{1} v_{2} \sigma_{1} v_{2} \underline{v_{1}} \\
& \cdot\left(v_{3} \ldots v_{i+1}\right)\left(v_{2} \ldots v_{i}\right)\left(v_{1} \ldots v_{i-1}\right) \\
= & \left(\underline{\left.v_{i-1} \ldots v_{1}\right)\left(v_{i} \ldots v_{1}\right)\left(v_{i+1} \ldots v_{2}\right)} \sigma_{1} v_{2} v_{1} \sigma_{1} v_{1} v_{2} \sigma_{1}\right. \\
& \cdot\left(\underline{\left.v_{2} \ldots v_{i+1}\right)\left(v_{1} \ldots v_{i}\right)\left(v_{1} \ldots v_{i-1}\right)}\right) \\
= & A .
\end{aligned}
$$

On the other hand, with similar manipulations we obtain

$$
\begin{aligned}
\sigma_{i+1} \sigma_{i} \sigma_{i+1}= & \cdots \\
= & \left(\underline{\left.v_{i} \ldots v_{1}\right)\left(v_{i+1} \ldots v_{2}\right)\left(v_{i+1} \ldots v_{3}\right)} \sigma_{1} v_{2} v_{1} \sigma_{1} v_{1} v_{2} \sigma_{1}\right. \\
& \cdot\left(\underline{\left.v_{3} \ldots v_{i+1}\right)\left(v_{2} \ldots v_{i+1}\right)\left(v_{1} \ldots v_{i}\right.}\right) \\
= & B .
\end{aligned}
$$

But

$$
\begin{aligned}
& \left(v_{i} \ldots v_{1}\right)\left(v_{i+1} v_{i} v_{i-1} \ldots v_{2}\right)\left(\underline{v_{i+1}} v_{i} \ldots v_{3}\right) \\
& =\left(v_{i} \ldots v_{1}\right) \underline{v_{i+1} v_{i} v_{i+1}}\left(v_{i-1} \ldots v_{2}\right)\left(v_{i} \ldots v_{3}\right) \\
& =\left(v_{i} v_{i-1} v_{i-2} \ldots v_{1}\right) \underline{v_{i}} v_{i+1} v_{i}\left(v_{i-1} \ldots v_{2}\right)\left(v_{i} \ldots v_{3}\right) \\
& =\underline{v_{i} v_{i-1} v_{i}}\left(v_{i-2} \ldots v_{1}\right)\left(v_{i+1} \ldots v_{2}\right)\left(v_{i} \ldots v_{3}\right) \\
& =v_{i-1} v_{i} v_{i-1}\left(v_{i-2} \ldots v_{1}\right)\left(\underline{v_{i+1}} v_{i} \ldots v_{2}\right)\left(v_{i} \ldots v_{3}\right) \\
& =\left(v_{i-1} v_{i} v_{i+1}\right)\left(v_{i-1} \ldots v_{1}\right)\left(v_{i} \ldots v_{2}\right)\left(v_{i} \ldots v_{3}\right) \\
& =\left(\underline{v_{i-1}} v_{i} v_{i+1}\right)\left(\underline{v_{i-2}} v_{i-1} v_{i}\right) \ldots\left(\underline{v_{1}} v_{2} v_{3}\right) v_{1} v_{2} \\
& =\left(v_{i-1} v_{i-2} \ldots v_{1}\right)\left(v_{i} v_{i-1} \ldots v_{2}\right)\left(v_{i+1} v_{i} \ldots v_{3}\right) \underline{v_{1}} v_{2} \\
& =\left(v_{i-1} \ldots v_{1}\right)\left(v_{i} \ldots v_{1}\right)\left(v_{i+1} \ldots v_{2}\right) \text {. }
\end{aligned}
$$

Hence, and by the symmetry of the underlined expressions in $A$ and $B$, we have shown that $B=A$.

LEMma 3. The braid relations $\sigma_{i} \sigma_{j}=\sigma_{j} \sigma_{i}$ for $i>1, i<j$ and $j \neq$ $i+1$ follow from the defining relations $(*)$, the virtual relations, the reduced relations $\sigma_{1} v_{j}=v_{j} \sigma_{1}$ of Lemma 1 , and the reduced relation

$$
\sigma_{1}\left(v_{2} v_{3} v_{1} v_{2} \sigma_{1} v_{2} v_{1} v_{3} v_{2}\right)=\left(v_{2} v_{3} v_{1} v_{2} \sigma_{1} v_{2} v_{1} v_{3} v_{2}\right) \sigma_{1} \text {. }
$$

Proof. Indeed, on the one hand we have

$$
\begin{aligned}
\sigma_{i} \sigma_{j} \stackrel{(*)}{=} & {\left[\left(v_{i-1} \ldots v_{1}\right)\left(v_{i} \ldots v_{2}\right) \sigma_{1}\left(v_{2} \ldots v_{i}\right)\left(v_{1} \ldots v_{i-1}\right)\right] } \\
& \cdot\left[\left(\underline{v_{j-1} \ldots v_{i+2} v_{i+1}} v_{i} \ldots v_{1}\right)\left(v_{j} \ldots v_{2}\right) \sigma_{1}\left(v_{2} \ldots v_{j}\right)\left(v_{1} \ldots v_{j-1}\right)\right]
\end{aligned}
$$




$$
\begin{aligned}
& =\left(v_{j-1} \ldots v_{i+2}\right)\left(v_{i-1} \ldots v_{1}\right)\left(v_{i} \ldots v_{2}\right) \sigma_{1}\left(v_{2} \ldots v_{i}\right) v_{i+1} \\
& \text { - } \left.\underline{\left(v_{1} \ldots v_{i-1}\right)\left(v_{i} \ldots v_{1}\right.}\right)\left(v_{j} \ldots v_{2}\right) \sigma_{1}\left(v_{2} \ldots v_{j}\right)\left(v_{1} \ldots v_{j-1}\right) \\
& \stackrel{(\dagger)}{=}\left(v_{j-1} \ldots v_{i+2}\right)\left(v_{i-1} \ldots v_{1}\right)\left(v_{i} \ldots v_{2}\right) \sigma_{1} \\
& \text { · } \left.\underline{\left(v_{2} \ldots v_{i} v_{i+1}\right)\left(v_{i} \ldots v_{2}\right.} v_{1} v_{2} \ldots v_{i}\right)\left(v_{j} \ldots v_{2}\right) \sigma_{1} \\
& \cdot\left(v_{2} \ldots v_{j}\right)\left(v_{1} \ldots v_{j-1}\right) \\
& \stackrel{(\dagger)}{=}\left(v_{j-1} \ldots v_{i+2}\right)\left(v_{i-1} \ldots v_{1}\right)\left(v_{i} \ldots v_{2}\right) \sigma_{1}\left(\underline{v_{i+1} \ldots v_{3}} v_{2} v_{3} \ldots v_{i+1}\right) \\
& \text { - }\left(v_{1} \ldots v_{i}\right)\left(\underline{v_{j} \ldots v_{i+3} v_{i+2}} v_{i+1} \ldots v_{2}\right) \sigma_{1}\left(v_{2} \ldots v_{j}\right)\left(v_{1} \ldots v_{j-1}\right) \\
& =\left(v_{j-1} \ldots v_{i+2}\right)\left(v_{j} \ldots v_{i+3}\right)\left(v_{i-1} \ldots v_{1}\right)\left(v_{i} \ldots v_{2}\right)\left(v_{i+1} \ldots v_{3}\right) \sigma_{1} \\
& \text {. }\left(v_{2} \ldots v_{i+1}\right) v_{i+2}\left(v_{1} \underline{v_{2} \ldots v_{i}}\right)\left(v_{i+1} v_{i} \ldots v_{2}\right) \sigma_{1}\left(v_{2} \ldots v_{j}\right)\left(v_{1} \ldots v_{j-1}\right) \\
& \stackrel{(\dagger)}{=}\left(v_{j-1} \ldots v_{i+2}\right)\left(v_{j} \ldots v_{i+3}\right)\left(v_{i-1} \ldots v_{1}\right)\left(v_{i} \ldots v_{2}\right)\left(v_{i+1} \ldots v_{3}\right) \sigma_{1} \\
& \cdot\left(v_{2} \ldots v_{i+2}\right) v_{1}\left(\underline{v_{i+1} \ldots v_{3}} v_{2} v_{3} \ldots v_{i+1}\right) \underline{\sigma_{1}}\left(v_{2} \ldots v_{j}\right)\left(v_{1} \ldots v_{j-1}\right) \\
& =\left(v_{j-1} \ldots v_{i+2}\right)\left(v_{j} \ldots v_{i+3}\right)\left(v_{i-1} \ldots v_{1}\right)\left(v_{i} \ldots v_{2}\right)\left(v_{i+1} \ldots v_{3}\right) \sigma_{1} \\
& \text {. }\left(v_{2} v_{3} \ldots v_{i+2}\right)\left(v_{i+1} \ldots v_{3}\right) v_{1} v_{2} \sigma_{1}\left(v_{3} \ldots v_{i+1}\right)\left(v_{2} \ldots v_{j}\right)\left(v_{1} \ldots v_{j-1}\right) \\
& \stackrel{(\dagger)}{=}\left(v_{j-1} \ldots v_{i+2}\right)\left(v_{j} \ldots v_{i+3}\right)\left(v_{i-1} \ldots v_{1}\right)\left(v_{i} \ldots v_{2}\right)\left(v_{i+1} \ldots v_{3}\right) \sigma_{1} \\
& \text { - } v_{2}\left(\underline{v_{i+2} \ldots v_{4}} v_{3} \underline{v_{4} \ldots v_{i+2}}\right) v_{1} v_{2} \sigma_{1}\left(v_{3} \ldots v_{i+1}\right)\left(v_{2} \ldots v_{j}\right)\left(v_{1} \ldots v_{j-1}\right) \\
& =\left(v_{j-1} \ldots v_{i+2}\right)\left(v_{j} \ldots v_{i+3}\right)\left(v_{i-1} \ldots v_{1}\right)\left(v_{i} \ldots v_{2}\right)\left(v_{i+1} \ldots v_{3}\right)\left(v_{i+2} \ldots v_{4}\right) \\
& \text { - } \sigma_{1} v_{2} v_{3} v_{1} v_{2} \sigma_{1}\left(v_{4} \ldots v_{i+2}\right)\left(v_{3} \ldots v_{i+1}\right)\left(v_{2} \ldots v_{j}\right)\left(v_{1} \ldots v_{j-1}\right) .
\end{aligned}
$$

But

$$
\begin{aligned}
\left(v_{4} \ldots v_{i+2}\right)\left(v_{3} \ldots v_{i+1}\right)\left(v_{2} \ldots v_{i} v_{i+1} \ldots v_{j}\right)\left(v_{1} \ldots v_{i-1} v_{i} \ldots v_{j-1}\right) & \stackrel{(\ddagger)}{=}\left(v_{4} v_{3} v_{2} v_{1}\right) \ldots\left(v_{i+2} \underline{v_{i+1} v_{i}} v_{i-1}\right)\left(\underline{v_{i+1}} v_{i+2} \ldots v_{j}\right)\left(v_{i} \ldots v_{j-1}\right) \\
= & \left(v_{4} v_{3} v_{2} v_{1}\right) \ldots\left(v_{i+1} v_{i} v_{i-1} v_{i-2}\right) \underline{v_{i}}\left(v_{i+2} v_{i+1} v_{i} v_{i-1}\right)\left(v_{i+2} \ldots v_{j}\right)\left(v_{i} \ldots v_{j-1}\right) \\
= & \ldots \\
= & v_{2}\left(v_{4} v_{3} v_{2} v_{1}\right) \ldots\left(v_{i+1} v_{i} v_{i-1} v_{i-2}\right)\left(v_{i+2} v_{i+1} \underline{v_{i} v_{i-1}}\right)\left(v_{i+2} \ldots v_{j}\right) \\
& \cdot\left(\underline{v_{i}} v_{i+1} \ldots v_{j-1}\right) \\
= & v_{2}\left(v_{4} v_{3} v_{2} v_{1}\right) \ldots\left(v_{i+1} v_{i} v_{i-1} v_{i-2}\right) \underline{v_{i-1}}\left(v_{i+2} v_{i+1} v_{i} v_{i-1}\right) \\
& \cdot\left(v_{i+2} \ldots v_{j}\right)\left(v_{i+1} \ldots v_{j-1}\right) \\
= & \ldots \\
= & \left.v_{2} v_{1}\left(v_{4} v_{3} v_{2} v_{1}\right) \ldots\left(v_{i+1} v_{i} v_{i-1} v_{i-2}\right) \underline{\left(v_{i+2} v_{i+1}\right.} v_{i} v_{i-1}\right)\left(\underline{v_{i+2}} v_{i+3} \ldots v_{j}\right) \\
& \cdot\left(v_{i+1} \ldots v_{j-1}\right)
\end{aligned}
$$




$$
\begin{aligned}
= & v_{2} v_{1}\left(v_{4} v_{3} v_{2} v_{1}\right) \ldots\left(v_{i+1} v_{i} v_{i-1} v_{i-2}\right) \underline{v_{i+1}}\left(v_{i+2} v_{i+1} v_{i} v_{i-1}\right) \\
& \cdot\left(v_{i+3} \ldots v_{j}\right)\left(v_{i+1} \ldots v_{j-1}\right) \\
= & \ldots \\
= & v_{2} v_{1} v_{3}\left(v_{4} v_{3} v_{2} v_{1}\right) \ldots\left(v_{i+1} v_{i} v_{i-1} v_{i-2}\right)\left(v_{i+2} \underline{v_{i+1} v_{i}} v_{i-1}\right)\left(v_{i+3} \ldots v_{j}\right) \\
& \cdot\left(\underline{v_{i+1}} v_{i+2} \ldots v_{j-1}\right) \\
= & \ldots \\
= & v_{2} v_{1} v_{3} v_{2}\left(v_{4} v_{3} v_{2} v_{1}\right) \ldots\left(v_{i+2} v_{i+1} v_{i} v_{i-1}\right)\left(v_{i+3} \ldots v_{j}\right)\left(v_{i+2} \ldots v_{j-1}\right) .
\end{aligned}
$$

Thus

$$
\begin{aligned}
& \sigma_{i} \sigma_{j}=\left(v_{j-1} \ldots v_{i+2}\right)\left(v_{j} \ldots v_{i+3}\right)\left(v_{i-1} \ldots v_{1}\right)\left(v_{i} \ldots v_{2}\right)\left(v_{i+1} \ldots v_{3}\right) \\
& \text { - }\left(v_{i+2} \ldots v_{4}\right) \underline{\sigma_{1}\left(v_{2} v_{3} v_{1} v_{2} \sigma_{1} v_{2} v_{1} v_{3} v_{2}\right)}\left(v_{4} v_{3} v_{2} v_{1}\right) \ldots\left(v_{i+2} v_{i+1} v_{i} v_{i-1}\right) \\
& \cdot\left(v_{i+3} \ldots v_{j}\right)\left(v_{i+2} \ldots v_{j-1}\right) \\
& =\left(v_{j-1} \ldots v_{i+2}\right)\left(v_{j} \ldots v_{i+3}\right)\left(v_{i-1} \ldots v_{1}\right)\left(v_{i} \ldots v_{2}\right)\left(v_{i+1} \ldots v_{3}\right)\left(v_{i+2} \ldots v_{4}\right) \\
& \cdot\left(\underline{v_{2}} v_{3} \underline{v_{1}} v_{2} \sigma_{1} v_{2} v_{1} v_{3} v_{2}\right) \sigma_{1} \underline{\left(v_{4} v_{3} v_{2} v_{1}\right) \ldots\left(v_{i+2} v_{i+1} v_{i} v_{i-1}\right)} \\
& \cdot\left(v_{i+3} \ldots v_{j}\right)\left(v_{i+2} \ldots v_{j-1}\right) \\
& \stackrel{(\ddagger)}{=}\left(v_{j-1} \ldots v_{i+2}\right)\left(v_{j} \ldots v_{i+3}\right)\left(v_{i-1} \ldots v_{1}\right)\left(v_{i} \ldots v_{2}\right)\left(v_{i+1} \ldots v_{3} v_{2} v_{1}\right) \\
& \cdot\left(v_{i+2} \ldots v_{4} v_{3} v_{2}\right) \sigma_{1} \underline{v_{2}} v_{1} \underline{v_{3}} v_{2} \sigma_{1}\left(\underline{v_{4} \ldots v_{i+2}}\right)\left(v_{3} \ldots v_{i+1}\right)\left(v_{2} \ldots v_{i}\right) \\
& \cdot\left(v_{1} \ldots v_{i-1}\right)\left(\underline{v_{i+3} \ldots v_{j}}\right)\left(v_{i+2} \ldots v_{j-1}\right) \\
& =\left(v_{j-1} \ldots v_{i+2}\right)\left(v_{j} \ldots v_{i+3}\right) \\
& \left.\cdot \underline{\left(v_{i-1} \ldots v_{1}\right)\left(v_{i} \ldots v_{2}\right)\left(v_{i+1} \ldots v_{3} v_{2} v_{1}\right)\left(v_{i+2} \ldots v_{4} v_{3} v_{2}\right.}\right) \\
& \cdot \sigma_{1}\left(v_{2} v_{3} v_{4} \ldots v_{i+2} v_{i+3} \ldots v_{j}\right) \\
& \cdot\left(v_{1} v_{2} \ldots v_{i+1} v_{i+2} \ldots v_{j-1}\right) \sigma_{1}\left(v_{2} \ldots v_{i}\right)\left(v_{1} \ldots v_{i-1}\right) \\
& \stackrel{(\ddagger)}{=}\left(v_{j-1} \ldots v_{i+2}\right)\left(v_{j} \ldots v_{i+3}\right)\left(v_{i-1} v_{i} v_{i+1} v_{i+2}\right) \ldots\left(v_{1} \underline{v_{2}} v_{3} v_{4}\right)\left(\underline{v_{2}} v_{1} v_{3} v_{2}\right) \\
& \text { - } \sigma_{1}\left(v_{2} \ldots v_{j}\right)\left(v_{1} \ldots v_{j-1}\right) \sigma_{1}\left(v_{2} \ldots v_{i}\right)\left(v_{1} \ldots v_{i-1}\right) \\
& =\cdots \\
& =\left(v_{j-1} \ldots v_{i+2}\right) v_{i+1}\left(v_{j} \ldots v_{i+3}\right)\left(v_{i-1} v_{i} v_{i+1} v_{i+2}\right) \ldots\left(\underline{v_{1} v_{2}} v_{3} v_{4}\right)\left(\underline{v_{1}} v_{3} v_{2}\right) \\
& \cdot \sigma_{1}\left(v_{2} \ldots v_{j}\right)\left(v_{1} \ldots v_{j-1}\right) \sigma_{1}\left(v_{2} \ldots v_{i}\right)\left(v_{1} \ldots v_{i-1}\right) \\
& =\cdots \\
& =\left(v_{j-1} \ldots v_{i+2}\right) v_{i+1} v_{i}\left(v_{j} \ldots v_{i+3}\right)\left(v_{i-1} v_{i} v_{i+1} v_{i+2}\right) \ldots\left(v_{1} v_{2} \underline{v_{3} v_{4}}\right)\left(\underline{v_{3}} v_{2}\right) \\
& \cdot \sigma_{1}\left(v_{2} \ldots v_{j}\right)\left(v_{1} \ldots v_{j-1}\right) \sigma_{1}\left(v_{2} \ldots v_{i}\right)\left(v_{1} \ldots v_{i-1}\right) \\
& =\cdots
\end{aligned}
$$




$$
\begin{aligned}
& =\left(v_{j-1} \ldots v_{i}\right)\left(v_{j} \ldots v_{i+3}\right) v_{i+2}\left(v_{i-1} v_{i} v_{i+1} v_{i+2}\right) \ldots\left(v_{1} \underline{v_{2} v_{3}} v_{4}\right) \underline{v_{2}} \\
& \text { - } \sigma_{1}\left(v_{2} \ldots v_{j}\right)\left(v_{1} \ldots v_{j-1}\right) \sigma_{1}\left(v_{2} \ldots v_{i}\right)\left(v_{1} \ldots v_{i-1}\right) \\
& =\cdots \\
& =\left(v_{j-1} \ldots v_{i}\right)\left(v_{j} \ldots v_{i+3}\right) v_{i+2} v_{i+1} \underline{\left(v_{i-1} v_{i} v_{i+1} v_{i+2}\right) \ldots\left(v_{1} v_{2} v_{3} v_{4}\right)} \\
& \cdot \sigma_{1}\left(v_{2} \ldots v_{j}\right)\left(v_{1} \ldots v_{j-1}\right) \sigma_{1}\left(v_{2} \ldots v_{i}\right)\left(v_{1} \ldots v_{i-1}\right) \\
& \stackrel{(\ddagger)}{=}\left(v_{j-1} \ldots v_{i}\right)\left(v_{j} \ldots v_{i+1}\right) \underline{\left(v_{i-1} \ldots v_{1}\right)}\left(v_{i} \ldots v_{2}\right)\left(v_{i+1} \ldots v_{3}\right)\left(v_{i+2} \ldots v_{4}\right) \\
& \text { - } \sigma_{1}\left(v_{2} \ldots v_{j}\right)\left(v_{1} \ldots v_{j-1}\right) \sigma_{1}\left(v_{2} \ldots v_{i}\right)\left(v_{1} \ldots v_{i-1}\right) \\
& =\left(v_{j-1} \ldots v_{1}\right)\left(v_{j} \ldots v_{2}\right) \sigma_{1}\left(v_{i+1} \ldots v_{3}\right)\left(v_{i+2} \ldots v_{4}\right)\left(v_{2} \ldots v_{j}\right)\left(v_{1} \ldots v_{j-1}\right) \\
& \text { - } \sigma_{1}\left(v_{2} \ldots v_{i}\right)\left(v_{1} \ldots v_{i-1}\right) \text {. }
\end{aligned}
$$

On the other hand, we have

$$
\begin{aligned}
\sigma_{j} \sigma_{i} \stackrel{(*)}{=} & \left(v_{j-1} \ldots v_{1}\right)\left(v_{j} \ldots v_{2}\right) \sigma_{1}\left(v_{2} \ldots v_{j}\right)\left(v_{1} \ldots v_{j-1}\right)\left(v_{i-1} \ldots v_{1}\right)\left(v_{i} \ldots v_{2}\right) \\
& \cdot \sigma_{1}\left(v_{2} \ldots v_{i}\right)\left(v_{1} \ldots v_{i-1}\right) .
\end{aligned}
$$

Therefore, in order that $\sigma_{i} \sigma_{j}=\sigma_{j} \sigma_{i}$ it suffices to show that the underlined expressions above are equal. Indeed, we have

$$
\begin{aligned}
\left(v_{i+1} \ldots v_{3}\right) & \left(v_{i+2} \ldots v_{4}\right)\left(\underline{v_{2}} v_{3} \ldots v_{j}\right)\left(v_{1} \ldots v_{j-1}\right) \\
= & \left.\left(v_{i+1} \ldots v_{3} v_{2}\right) \underline{\left(v_{i+2} \ldots v_{4}\right)\left(v_{3} v_{4} \ldots v_{i+2}\right.} v_{i+3} \ldots v_{j}\right)\left(v_{1} \ldots v_{j-1}\right) \\
\stackrel{(\dagger)}{=} & \left(\underline{\left.v_{i+1} \ldots v_{3} v_{2}\right)\left(v_{3} \ldots v_{i+1}\right.} v_{i+2} v_{i+1} \ldots v_{3}\right)\left(v_{i+3} \ldots v_{j}\right)\left(v_{1} \ldots v_{j-1}\right) \\
\stackrel{(\dagger)}{=} & \left(\underline{v_{2} \ldots v_{i} v_{i+1}} v_{i} \ldots v_{2}\right)\left(\underline{v_{i+2}} v_{i+1} \ldots v_{3}\right)\left(\underline{v_{i+3} \ldots v_{j}}\right)\left(\underline{v_{1}} v_{2} \ldots v_{j-1}\right) \\
= & \left(v_{2} \ldots v_{i+1} v_{i+2} v_{i+3} \ldots v_{j}\right)\left(v_{i} \ldots v_{2}\right) v_{1} \\
& \cdot\left(\underline{\left.v_{i+1} \ldots v_{3}\right)\left(v_{2} v_{3} \ldots v_{i+1}\right.} v_{i+2} \ldots v_{j-1}\right) \\
\stackrel{(\dagger)}{=} & \left(v_{2} \ldots v_{j}\right)\left(\underline{\left.v_{i} \ldots v_{2} v_{1}\right)\left(v_{2} \ldots v_{i}\right.} v_{i+1} v_{i} \ldots v_{2}\right)\left(v_{i+2} \ldots v_{j-1}\right) \\
\stackrel{\dagger}{=} & \left(v_{2} \ldots v_{j}\right)\left(\underline{v_{1} \ldots v_{i-1} v_{i}} v_{i-1} \ldots v_{1}\right)\left(\underline{v_{i+1}} v_{i} \ldots v_{2}\right)\left(\underline{v_{i+2} \ldots v_{j-1}}\right) \\
= & \left(v_{2} \ldots v_{j}\right)\left(v_{1} \ldots v_{j-1}\right)\left(v_{i-1} \ldots v_{1}\right)\left(v_{i} \ldots v_{2}\right) .
\end{aligned}
$$

By Lemmas 1, 2, and 3 the proof of Theorem 2 is now concluded.

4. A reduced presentation for the flat virtual braid group. The flat virtual braids were introduced in [17]. As with the virtual braids, the set of flat virtual braids on $n$ strands forms a group, the flat virtual braid group, denoted $F V_{n}$. The generators of $F V_{n}$ are the virtual crossings $v_{1}, \ldots, v_{n-1}$ 


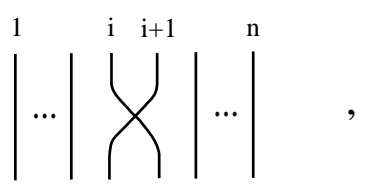

$c_{i}$

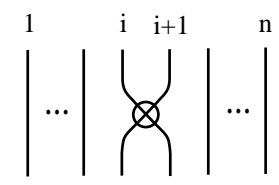

$v_{i}$

Fig. 19. The generators of $F V_{n}$

and the flat crossings $c_{1}, \ldots, c_{n-1}$, which - as already said in 1.1 - can be seen as immersed crossings. See Figure 19.

So, flat crossings and virtual crossings both represent geometrically the generators of the symmetric group $S_{n}$. But the mixed relations between them are not symmetric (see below). In fact, the flat virtual braid group is the quotient of the virtual braid group $V B_{n}$ modulo the relations $\sigma_{i}^{2}=1$ for all $i$. Thus, $F V_{n}$ is the free product of two copies of $S_{n}$, modulo the set of mixed relations specified below. Note that $F V_{2}=S_{2} * S_{2}$ (no extra relations), and it is infinite.

Recall that in Section 1.1 we discussed flat virtual knots and links, and that we pointed out that this category is equivalent to the category of virtual strings developed in [26]. Just so, the flat virtual braids are the appropriate theory of braids for the category of virtual strings. Every virtual string is the closure of a flat virtual braid.

The virtual generators satisfy among themselves the virtual relations. Similarly, the flat generators satisfy among themselves the following flat relations:

$$
\begin{aligned}
c_{i}^{2} & =1, \\
c_{i} c_{i+1} c_{i} & =c_{i+1} c_{i} c_{i+1}, \\
c_{i} c_{j} & =c_{j} c_{i} \quad \text { for } j \neq i \pm 1 .
\end{aligned}
$$

The mixed flat relations between flat and virtual generators are as follows:

$$
\begin{aligned}
c_{i} v_{j} & =v_{j} c_{i} \quad \text { for } j \neq i \pm 1, \\
v_{i} c_{i+1} v_{i} & =v_{i+1} c_{i} v_{i+1} .
\end{aligned}
$$

The second mixed relation will be called the special detour flat relation and it is illustrated in Figure 9. Then, as for the virtual braids, we have for the flat crossings the inductive defining relations $c_{i+1}=v_{i} v_{i+1} c_{i} v_{i+1} v_{i}$, which leads to the defining relations

$$
c_{i+1}:=\left(v_{i} \ldots v_{2} v_{1}\right)\left(v_{i+1} \ldots v_{3} v_{2}\right) c_{1}\left(v_{2} v_{3} \ldots v_{i+1}\right)\left(v_{1} v_{2} \ldots v_{i}\right)
$$

for $i=2, \ldots, n-1$. In terms of flat braid diagrams, this relation is the flat braid detour move of the strands $1,2, \ldots, i-1$ around the flat crossing $c_{i}$. In complete analogy to the virtual braid group we now have the following: 
THEOREM 3. The flat virtual braid group $F V_{n}$ has the following reduced presentation:

$$
\begin{array}{r}
F V_{n}=\left\langle c_{1}, v_{1}, \ldots, v_{n-1}\right| v_{i} v_{i+1} v_{i}=v_{i+1} v_{i} v_{i+1}, v_{i} v_{j}=v_{j} v_{i}, j \neq i \pm 1, \\
c_{1}^{2}=1, v_{i}^{2}=1,1 \leq i \leq n-1, c_{1} v_{j}=v_{j} c_{1}, j>2, \\
\left(v_{1} c_{1} v_{1}\right)\left(v_{2} c_{1} v_{2}\right)\left(v_{1} c_{1} v_{1}\right)=\left(v_{2} c_{1} v_{2}\right)\left(v_{1} c_{1} v_{1}\right)\left(v_{2} c_{1} v_{2}\right), \\
\left.c_{1}\left(v_{2} v_{3} v_{1} v_{2} c_{1} v_{2} v_{1} v_{3} v_{2}\right)=\left(v_{2} v_{3} v_{1} v_{2} c_{1} v_{2} v_{1} v_{3} v_{2}\right) c_{1}\right\rangle .
\end{array}
$$

5. Other categories. Welded braids were introduced in [7]. They satisfy the same isotopy relations as the virtuals, but for welded braids one also allows one of the two forbidden moves, the move $\left(F_{1}\right)$ of Figure 4 , which contains an over arc and one virtual crossing. One can consider welded knots and links in this way, and the explanation for the choice of moves lies in the fact that the first forbidden move preserves the combinatorial fundamental group. This is not true for the other forbidden move $\left(F_{2}\right)$. The corresponding welded braid group on $n$ strands, $W B_{n}$, has the same generators and relations as the virtual braid group, but with the extra relations

$$
v_{i} \sigma_{i+1} \sigma_{i}=\sigma_{i+1} \sigma_{i} v_{i+1} .
$$

Figure 4 illustrates a variant of this relation. Just as in the virtual braid group, the braiding generators $\sigma_{2}, \ldots, \sigma_{n-1}$ of the welded braid group can be written in terms of $\sigma_{1}$ and the welded generators $v_{1}, \ldots, v_{n-1}$. We can then obtain a reduced presentation for $W B_{n}$ with generators

$$
\left\{\sigma_{1}, v_{1}, \ldots, v_{n-1}\right\}
$$

and the defining relations

$$
\sigma_{i+1}:=\left(v_{i} \ldots v_{2} v_{1}\right)\left(v_{i+1} \ldots v_{3} v_{2}\right) \sigma_{1}\left(v_{2} v_{3} \ldots v_{i+1}\right)\left(v_{1} v_{2} \ldots v_{i}\right) .
$$

By the box detour trick (see Remark 2) we can easily reduce the set of extra relations $\left(F_{1}\right)$ to the basic relation

$$
v_{1} \sigma_{2} \sigma_{1}=\sigma_{2} \sigma_{1} v_{2}
$$

which with the substitution $\sigma_{2}=v_{1} v_{2} \sigma_{1} v_{2} v_{1}$ is equivalent to the relation

$$
v_{2} \sigma_{1} v_{2} v_{1} \sigma_{1}=v_{1} v_{2} \sigma_{1} v_{2} v_{1} \sigma_{1} v_{2} .
$$

Thus we have:

THEOREM 4. The welded braid group $W B_{n}$ has the following reduced presentation: 
$W B_{n}=\left\langle\sigma_{1}, v_{1}, \ldots, v_{n-1}\right| v_{i} v_{i+1} v_{i}=v_{i+1} v_{i} v_{i+1}, v_{i} v_{j}=v_{j} v_{i}, j \neq i \pm 1$

$$
\begin{gathered}
v_{i}^{2}=1,1 \leq i \leq n-1, \sigma_{1} v_{j}=v_{j} \sigma_{1}, j>2, \\
\left(v_{1} \sigma_{1} v_{1}\right)\left(v_{2} \sigma_{1} v_{2}\right)\left(v_{1} \sigma_{1} v_{1}\right)=\left(v_{2} \sigma_{1} v_{2}\right)\left(v_{1} \sigma_{1} v_{1}\right)\left(v_{2} \sigma_{1} v_{2}\right), \\
v_{1}\left(v_{2} \sigma_{1} v_{2} v_{1} \sigma_{1}\right)=\left(v_{2} \sigma_{1} v_{2} v_{1} \sigma_{1}\right) v_{2}, \\
\left.\sigma_{1}\left(v_{2} v_{3} v_{1} v_{2} \sigma_{1} v_{2} v_{1} v_{3} v_{2}\right)=\left(v_{2} v_{3} v_{1} v_{2} \sigma_{1} v_{2} v_{1} v_{3} v_{2}\right) \sigma_{1}\right\rangle .
\end{gathered}
$$

Note now that the relations $\left(F_{1}\right)$ can be regarded as a way of detouring sequences of classical crossings over welded crossings, via the inductive relations

$$
v_{i+1}=\sigma_{i}^{-1} \sigma_{i+1}^{-1} v_{i} \sigma_{i+1} \sigma_{i}
$$

which, by induction, lead to the defining relations

$(* *) \quad v_{i+1}:=\left(\sigma_{i}^{-1} \ldots \sigma_{i}^{-1}\right)\left(\sigma_{i+1}^{-1} \ldots \sigma_{2}^{-1}\right) v_{1}\left(\sigma_{2} \ldots \sigma_{i+1}\right)\left(\sigma_{1} \ldots \sigma_{i}\right)$

for $i=1, \ldots, n-2$. By the box detour trick we reduce the relations involving welded generators. For example, the welded relations reduce to the following two basic ones:

$$
v_{1} v_{2} v_{1}=v_{2} v_{1} v_{2} \quad \text { and } \quad v_{1} v_{3}=v_{3} v_{1}
$$

Thus, we obtain the following reduced presentation for $W B_{n}$ with a single welded generator.

THEOREM 5. The welded braid group $W B_{n}$ has the following reduced presentation:

$$
\begin{aligned}
W B_{n}=\left\langle v_{1}, \sigma_{1}, \ldots, \sigma_{n-1}\right| \sigma_{i} \sigma_{i+1} \sigma_{i}=\sigma_{i+1} \sigma_{i} \sigma_{i+1}, \sigma_{i} \sigma_{j}=\sigma_{j} \sigma_{i}, j \neq i \pm 1 \\
v_{1}^{2}=1, v_{1} \sigma_{j}=\sigma_{j} v_{1}, j>2 \\
\left(\sigma_{1} v_{1} \sigma_{1}^{-1}\right)\left(\sigma_{2}^{-1} v_{1} \sigma_{2}\right)\left(\sigma_{1} v_{1} \sigma_{1}^{-1}\right)=\left(\sigma_{2}^{-1} v_{1} \sigma_{2}\right)\left(\sigma_{1} v_{1} \sigma_{1}^{-1}\right)\left(\sigma_{2}^{-1} v_{1} \sigma_{2}\right), \\
\left.v_{1}\left(\sigma_{2}^{-1} \sigma_{1}^{-1} \sigma_{3}^{-1} \sigma_{2}^{-1} v_{1} \sigma_{2} \sigma_{3} \sigma_{1} \sigma_{2}\right)=\left(\sigma_{2}^{-1} \sigma_{1}^{-1} \sigma_{3}^{-1} \sigma_{2}^{-1} v_{1} \sigma_{2} \sigma_{3} \sigma_{1} \sigma_{2}\right) v_{1}\right\rangle .
\end{aligned}
$$

Another generalization of the virtual braid group is obtained by adding both types of forbidden moves (recall Figure 4). We call this the unrestricted virtual braid group, denoted $U B_{n}$. It is known that any classical knot can be unknotted in the virtual category if we allow both forbidden moves [15, 24]. Nevertheless, linking phenomena still remain. The unrestricted braid group itself is non-trivial, deserving further study.

By adding both types of forbidden moves:

$$
v_{i} \sigma_{i+1} \sigma_{i}=\sigma_{i+1} \sigma_{i} v_{i+1} \quad\left(F_{1}\right) \quad \text { and } \quad \sigma_{i} \sigma_{i+1} v_{i}=v_{i+1} \sigma_{i} \sigma_{i+1}
$$

and using the defining relations $(*)$ we obtain a reduced presentation for 
$U B_{n}$ with generators $\sigma_{1}, v_{1}, \ldots, v_{n-1}$, which is in fact a quotient of the corresponding reduced presentation of $W B_{n}$ by the second forbidden move:

$$
\sigma_{1} \sigma_{2} v_{1}=v_{2} \sigma_{1} \sigma_{2}
$$

THEOREM 6. The unrestricted virtual braid group has the following reduced presentation:

$$
\begin{gathered}
U B_{n}=\left\langle\sigma_{1}, v_{1}, \ldots, v_{n-1}\right| v_{i} v_{i+1} v_{i}=v_{i+1} v_{i} v_{i+1}, v_{i} v_{j}=v_{j} v_{i}, j \neq i \pm 1, \\
v_{i}^{2}=1,1 \leq i \leq n-1, \sigma_{1} v_{j}=v_{j} \sigma_{1}, j>2, \\
\left(v_{1} \sigma_{1} v_{1}\right)\left(v_{2} \sigma_{1} v_{2}\right)\left(v_{1} \sigma_{1} v_{1}\right)=\left(v_{2} \sigma_{1} v_{2}\right)\left(v_{1} \sigma_{1} v_{1}\right)\left(v_{2} \sigma_{1} v_{2}\right), \\
v_{1}\left(v_{2} \sigma_{1} v_{2} v_{1} \sigma_{1}\right)=\left(v_{2} \sigma_{1} v_{2} v_{1} \sigma_{1}\right) v_{2} \\
\left(\sigma_{1} v_{1} v_{2} \sigma_{1} v_{2}\right) v_{1}=v_{2}\left(\sigma_{1} v_{1} v_{2} \sigma_{1} v_{2}\right), \\
\\
\left.\sigma_{1}\left(v_{2} v_{3} v_{1} v_{2} \sigma_{1} v_{2} v_{1} v_{3} v_{2}\right)=\left(v_{2} v_{3} v_{1} v_{2} \sigma_{1} v_{2} v_{1} v_{3} v_{2}\right) \sigma_{1}\right\rangle .
\end{gathered}
$$

Just as in the case of welded braids, we can also give a reduced presentation with one virtual generator and $n-1$ braiding generators. For unrestricted virtual braids, there are two possible such reduced presentations, depending upon using either the first or the second forbidden move in performing the detour substitutions. In the $\left(F_{1}\right)$ case the defining relations are given by $(* *)$ and, thus, the reduced presentation is a quotient of the corresponding presentation of the welded braid group by the relation

$$
\sigma_{1} \sigma_{2} v_{1} \sigma_{2}^{-1} \sigma_{1}^{-1}=\sigma_{1}^{-1} \sigma_{2}^{-1} v_{1} \sigma_{2} \sigma_{1} .
$$

Since in this presentation $v_{2}$ is defined via $\left(F_{1}\right)$ in terms of $v_{1}$, the reader will note that the transcription of this last relation appears to be a mixture of $\left(F_{1}\right)$ and $\left(F_{2}\right)$. Similarly we could have started with $\left(F_{2}\right)$ and obtained first an analogue of the welded braid group and then, adding $\left(F_{1}\right)$, obtained another reduced presentation of the unrestricted virtual braid group.

Finally, we define the flat unrestricted braid group, denoted $F U_{n}$, to be the quotient of the flat virtual braid group $F V_{n}$ (see Theorem 3) by the forbidden moves of $F V_{n}$ (see Figure 9):

$$
c_{i} c_{i+1} v_{i}=v_{i+1} c_{i} c_{i+1} .
$$

Note that for the flat virtual braid group there is only one type of forbidden move.

THEOREM 7. The flat unrestricted braid group $F U_{n}$ has the following reduced presentation: 


$$
\begin{gathered}
F U_{n}=\left\langle c_{1}, v_{1}, \ldots, v_{n-1}\right| v_{i} v_{i+1} v_{i}=v_{i+1} v_{i} v_{i+1}, v_{i} v_{j}=v_{j} v_{i}, j \neq i \pm 1 \\
c_{1}^{2}=1, v_{i}^{2}=1,1 \leq i \leq n-1, c_{1} v_{j}=v_{j} c_{1}, j>2, \\
\left(v_{1} c_{1} v_{1}\right)\left(v_{2} c_{1} v_{2}\right)\left(v_{1} c_{1} v_{1}\right)=\left(v_{2} c_{1} v_{2}\right)\left(v_{1} c_{1} v_{1}\right)\left(v_{2} c_{1} v_{2}\right) \\
v_{1}\left(v_{2} c_{1} v_{2} v_{1} c_{1}\right)=\left(v_{2} c_{1} v_{2} v_{1} c_{1}\right) v_{2} \\
\left.c_{1}\left(v_{2} v_{3} v_{1} v_{2} c_{1} v_{2} v_{1} v_{3} v_{2}\right)=\left(v_{2} v_{3} v_{1} v_{2} c_{1} v_{2} v_{1} v_{3} v_{2}\right) c_{1}\right\rangle .
\end{gathered}
$$

REMARK 3. Note that the flat unrestricted braid group $F U_{n}$ is a free product with amalgamation of two copies of the symmetric group $S_{n}$. An unreduced presentation of $F U_{n}$ can be configured to be symmetric with respect to the roles of the generators $c_{i}$ and $v_{i}$. As a result there is another reduced presentation that can be obtained from the reduced presentation above by interchanging the roles of $v_{i}$ and $c_{i}$.

REMARK 4. Note that the flat unrestricted braid group $F U_{n}$ is also a quotient of the welded braid group $W B_{n}$ (see Theorem 4), obtained by setting all the squares of the braiding generators equal to 1 . Thus there is a surjective homomorphism from $W B_{n}$ to $F U_{n}$. This homomorphism is a direct analogue of the standard homomorphism from $B_{n}$ to the symmetric group $S_{n}$. Figure 20 gives a commutative diagram of these relationships. Note that all structures map eventually to the symmetric group $S_{n}$. In the case of the virtual braids and their quotients, this map to the symmetric group takes the same value on virtual generators $v_{i}$ and braiding generators $\sigma_{i}$. The intermediate mappings to $F V_{n}$ and $F U_{n}$ preserve these differences.

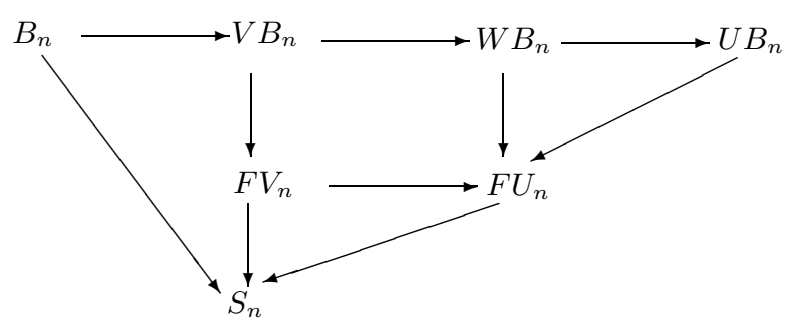

Fig. 20. A diagram of relationships

6. Welded braids and tubes in four-space. The welded braid group $W B_{n}$ can be interpreted as the fundamental group of the configuration space of $n$ disjoint circles trivially embedded in three-dimensional space $\mathbb{R}^{3}$. This group (the so-called motion group of disjoint circles) can, in turn, be interpreted as a braid group of tubes embedded in $\mathbb{R}^{3} \times \mathbb{R}=\mathbb{R}^{4}$. These braided tubes in four-space are generated by two types of elementary braiding. In Figure 21, we show diagrams that can be interpreted as immersions of tubes 

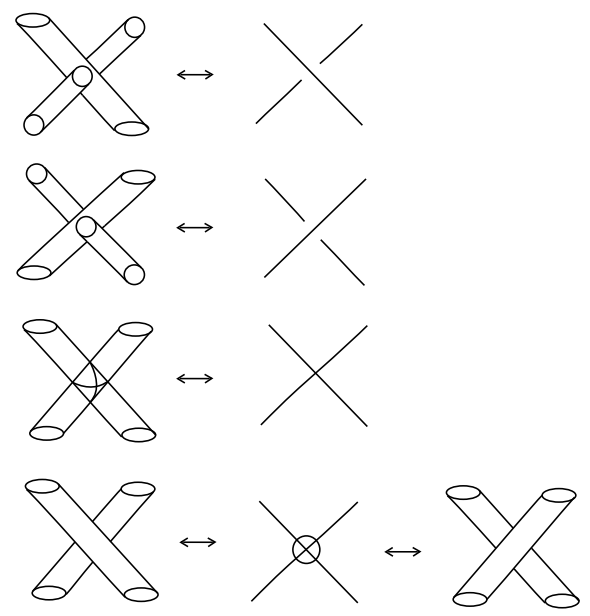

Fig. 21. Tubular correspondences

in three-space. Each such immersion is a projection of a corresponding embedding in four-space. The first two diagrams of Figure 21 each illustrate a tube passing through another tube. When tube $A$ passes through tube $B$ we make a corresponding classical braiding crossing with arc $A$ passing under $\operatorname{arc} B$. The four-dimensional interpretation of tube $A$ passing through tube $B$ is that: As one looks at the levels of intersection with $\mathbb{R}^{3} \times t$ for different values of $t$, one sees two circles $A(t)$ and $B(t)$. As the variable $t$ increases, the $A(t)$ circle (always disjointly embedded from the $B(t)$ circle) moves through the $B(t)$ circle. This process is illustrated in Figure 22 .

While the classical crossing in a welded braid diagram corresponds to a genuine braiding of the tubes in four-space (as described above), the virtual crossing corresponds to tubes that do not interact in the immersion representation (see again Figure 21). These non-interacting tubes can pass over or under each other, as these local projections correspond to equivalent embeddings in four-space.

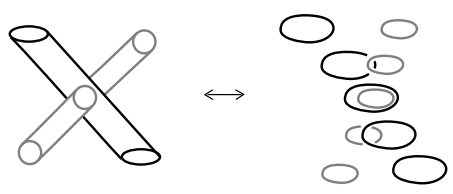

Fig. 22. Braiding of circles

It is an interesting exercise to verify that the moves in the welded braid group each induce equivalences of the corresponding tubular braids in fourspace. In particular, the move $\left(F_{1}\right)$ induces such an isotopy, while the forbidden move $\left(F_{2}\right)$ does not. For more on this subject, the reader can consult 
[25] and also [18] and the references therein. The basic idea for this correspondence is due to Satoh [25], where torus embeddings in four-space are associated with virtual knot diagrams.

Consider now the surjection $W B_{n} \rightarrow F U_{n}$ from the welded braid group to the flat unrestricted braids. Flat unrestricted braids can be represented by immersions of tubes in three-space as illustrated also in Figure 21. There we have indicated a decorated immersion of two intersecting tubes as the correspondent of the flat classical crossing in $F U_{n}$. One must specify the rules for handling these immersions in order to obtain the correspondence. We omit that discussion here, but point out the interest in having a uniform context for the surjection of the welded braids to the flat unrestricted braids. The flat unrestricted braids carry the distinction between braided flat and welded flat crossings and otherwise keep track of the relative permutations of these two types of crossing.

Acknowledgements. Much of this effort was sponsored by the Defense Advanced Research Projects Agency (DARPA) and Air Force Research Laboratory, Air Force Materiel Command, USAF, under agreement F30602-01-2-05022. The U.S. Government is authorized to reproduce and distribute reprints for Government purposes notwithstanding any copyright annotations thereon. The views and conclusions contained herein are those of the author and should not be interpreted as necessarily representing the official policies or endorsements, either expressed or implied, of the Defense Advanced Research Projects Agency, the Air Force Research Laboratory, or the U.S. Government. It gives the first author great pleasure to acknowledge support from NSF Grant DMS-0245588, and to give thanks to the University of Waterloo and the Perimeter Institute in Waterloo, Canada for their hospitality during the preparation of this research.

\section{References}

[1] J. W. Alexander, A lemma on systems of knotted curves, Proc. Nat. Acad. Sci. U.S.A. 9 (1923), 93-95.

[2] J. C. Baez, Link invariants of finite type and perturbation theory, Lett. Math. Phys. 26 (1992), 43-51.

[3] J. Birman, New points of view in knot theory, Bull. Amer. Math. Soc. 28 (1993), $253-287$.

[4] H. Brunn, Über verknotete Curven, Verh. des intern. Math. Congr. 1 (1897), 256259.

[5] J. S. Carter, S. Kamada and M. Saito, Stable equivalence of knots on surfaces and virtual knot cobordisms, J. Knot Theory Ramifications 11 (2002), 311-320.

[6] H. Dye and L. H. Kauffman, Minimal surface representations of virtual knots and links, arXiv:math.AT/0401035. 
[7] R. Fenn, R. Rimanyi and C. Rourke, The braid permutation group, Topology 36 (1997), 123-135.

[8] R. Fenn, D. Rolfsen and J. Zhu, Centralizers in the braid group and singular braid monoid, Enseign. Math. (2) 42 (1996), 75-96.

[9] M. Goussarov, M. Polyak and O. Viro, Finite type invariants of classical and virtual knots, Topology 39 (2000), 1045-1068.

[10] D. Hrencecin, On filamentations and virtual knot invariants, Ph.D. thesis, Univ. of Illinois at Chicago, 2001.

[11] D. Hrencecin and L. H. Kauffman, On filamentations and virtual knots, Topology Appl. 134 (2003), 23-52.

[12] T. Kadokami, Detecting non-triviality of virtual links, J. Knot Theory Ramifications 12 (2003), 781-803.

[13] S. Kamada, Braid presentation of virtual knots and welded knots, preprint, 2000.

[14] N. Kamada and S. Kamada, Abstract link diagrams and virtual knots, J. Knot Theory Ramifications 9 (2000), 93-109.

[15] T. Kanenobu, Forbidden moves unknot a virtual knot, ibid. 10 (2001), 89-96.

[16] L. H. Kauffman, Virtual knot theory, European J. Combin. 20 (1999), 663-690.

[17] -, A survey of virtual knot theory, in: Knots in Hellas '98, World Sci., 2000, 143-202.

[18] -, Detecting virtual knots, Atti Sem. Mat. Fis. Univ. Modena 49 (2001), suppl., 241-282.

[19] L. Kauffman and P. Vogel, Link polynomials and a graphical calculus, J. Knot Theory Ramifications 1 (1992), 59-104.

[20] T. Kishino and S. Satoh, A note on non-classical virtual knots, ibid. 13 (1004), 845-856.

[21] G. Kuperberg, What is a virtual link?, Algebr. Geom. Topol. 3 (2003), 587-591.

[22] S. Lambropoulou and C. P. Rourke, Markov's theorem in three-manifolds, Topology Appl. 78 (1997), 95-122.

[23] V. Manturov, On the recognition of virtual braids, Zap. Nauchn. Sem. POMI 8 (2003), 267-286 (in Russian).

[24] S. Nelson, Unknotting virtual knots with Gauss diagram forbidden moves, J. Knot Theory Ramifications 10 (2001), 931-935.

[25] S. Satoh, Virtual knot presentation of ribbon torus-knots, ibid. 9 (2000), 531-542.

[26] V. Turaev, Virtual strings and their cobordisms, arXiv.math.GT/0311185.

[27] V. V. Vershinin, On homology of virtual braids and Burau representation, J. Knot Theory Ramifications 10 (2001), 795-812.

[28] —, On the singular braid monoid, arXiv:Math.GR/0309339, 2003.

Department of Mathematics,

Statistics and Computer Science

University of Illinois at Chicago

851 South Morgan St.

Chicago, IL 60607-7045, U.S.A.

E-mail: kauffman@uic.edu

URL: www.math.uic.edu/ ${ }^{\sim}$ kauffman
Department of Mathematics National Technical University of Athens Zografou Campus, GR-157 80 Athens, Greece E-mail: sofia@math.ntua.gr URL: http://www.math.ntua.gr/ ${ }^{\sim}$ sofia and Laboratoire de Mathématiques Nicolas Oresme Université de Caen F-14032 Caen Cedex, France E-mail: sofia@math.unicaen.fr 